2020-04-01

\title{
Water-wave interaction with submerged porous elastic disks
}

\author{
Zheng, Siming
}

http://hdl.handle.net/10026.1/15576

10.1063/5.0006119

Physics of Fluids

AIP Publishing

All content in PEARL is protected by copyright law. Author manuscripts are made available in accordance with publisher policies. Please cite only the published version using the details provided on the item record or document. In the absence of an open licence (e.g. Creative Commons), permissions for further reuse of content should be sought from the publisher or author. 


\title{
Water-wave interaction with submerged porous elastic disks
}

\author{
Siming Zheng ${ }^{\mathrm{a}, \mathrm{b}}$, Michael H. Meylan ${ }^{\mathrm{c}}$, Deborah Greaves ${ }^{\mathrm{b}}$, Gregorio Iglesias ${ }^{\mathrm{d}, \mathrm{b}}$ \\ a State Key Laboratory of Hydraulic Engineering Simulation and Safety, Tianjin University, Tianjin \\ 300072, China \\ $b$ School of Engineering, Computing and Mathematics, University of Plymouth, Drake Circus, \\ Plymouth PL4 8AA, UK \\ c School of Mathematical and Physical Sciences, The University of Newcastle, Callaghan 2308, \\ Australia \\ $d$ Centre for Marine and Renewable Energy Ireland (MaREI), Environmental Research Institute \& \\ School of Engineering, University College Cork, Ireland
}

Cite as: Phys. Fluids 32, 047106 (2020); https://doi.org/10.1063/5.0006119

Submitted: 27 February 2020

Accepted: 01 April 2020

Published Online: 17 April 2020 
Water-wave interaction with submerged porous elastic disks

S. Zheng (郑思明), ${ }^{1,2, a)}$ M. H. Meylan, ${ }^{3}$ D. Greaves,${ }^{2}$ and G. Iglesias ${ }^{4,2}$

${ }^{1)}$ State Key Laboratory of Hydraulic Engineering Simulation and Safety, Tianjin University, Tianjin 300072, China

${ }^{2)}$ School of Engineering, Computing and Mathematics, University of Plymouth, Drake Circus, Plymouth PLA 8AA, UK

${ }^{3)}$ School of Mathematical and Physical Sciences, The University of Newcastle, Callaghan 2308, Australia

${ }^{4)}$ MaREI, Environmental Research Institute \& School of Engineering, University College Cork, Ireland

(Dated: 17 April 2020)

Hydroelastic interaction between water-waves and submerged porous elastic disks of negligible thickness in water of finite depth is investigated under the assumption of small amplitude water wave motion and structural response. The disks are either simply supported or clamped at their edges. Wave power can be absorbed/dissipated by the disks due to their porosity. A theoretical model based on the linear potential flow theory and eigenfunction matching method is developed to solve the wave scattering problem of the submerged disks. An indirect method, employing Kochin functions, is derived based on Green's theorem to evaluate the wave power absorption/dissipation, and it produces accurate results at a lower computational cost than the conventional method. This theoretical model is applied to perform a multi-parameter study on the performance of a single submerged porous elastic disk, and an array of disks as well, particularly in terms of nearfield wave motion, disk deflection, far-field scattering coefficient, and wave power absorption/dissipation. Deploying multiple disks in an array is found to be a more promising approach for wave power absorption/dissipation compared to enlarging the area of a single disk. (doi: 10.1063/5.0006119)

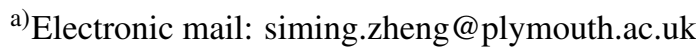




\section{INTRODUCTION}

Among a large variety of marine structures, some of them can be classified as a horizontal plate/disk, e.g., sea ice floes (Squire, 2020; Meylan and Squire, 1996) and very large floating structures, such as airports and artificial islands (Lamas-Pardo, Iglesias, and Carral, 2015). The horizontal dimensions of these structures are vast, while the vertical dimension is small, making it difficult to carry out experimental investigations (Zhao, Zhang, and Huang, 2007). Therefore, theoretical and numerical methods have been extensively applied to study water-wave interaction of floating plates/discs.

Meylan and Squire (1996) introduced a theoretical model to reproduce the behaviour of a circular flexible ice floe subjected to long-crested sea waves. Sahoo, Yip, and Chwang (2001) studied the hydroelastics of a floating semi-infinite elastic plate in finite water depth. Compared to a free edge and a simply supported edge, a built-in edge was found to produce more significant wave reflection and the minimum wave transmission. Meylan, Bennetts, and Peter (2017) developed a theoretical model and a numerical model to study wave-water scattering from a floating porous elastic disc. The conceptual model was extended recently by Zheng et al. (2020) to investigate a floating porous elastic plate of arbitrary shape. Oblique water-wave scattering by a floating flexible porous plate in infinite depth was studied by Koley, Mondal, and Sahoo (2018) with the employment of a Fredholm integral equation technique. More detailed literature review regarding the investigation of floating plates/discs can be found in Squire (2008, 2020) and Zheng et al. (2020).

Submerged plates are another kind of marine structure comparable to floating plates. Compared with floating structures, the submerged plates have a reduced environmental and visual impact, and are less exposed to severe wave breaking. Lower construction cost of submerged structures is another advantage (Ahmadian, 2016). The benefits of submerged structures over floating ones make them particularly attractive for protecting natural beaches and exploiting marine renewable energy. Indeed, extensive studies have been performed on the water-wave interaction with submerged plates.

Yu and Chwang (1993) studied the scattering of regular waves by a circular horizontal solid impermeable disk submerged at a finite depth beneath the water level by means of a theoretical model. An effect of wave focusing around the rear of the disk was demonstrated. The wave radiation problem of a heaving submerged horizontal disk was reduced by Martin and Farina (1997) 
to solving a hypersingular integral equation. In their model, this equation was further reduced to one-dimensional Fredholm integral equations of the second kind. Later, Farina and Martin (1998) developed an expansion-collocation method to solve two-dimensional (2D) hypersingular integral equations for wave radiation and scattering problems of a submerged disk. Porter (2015) proposed a model based an integral transform approach and a Galerkin method, and described the application of the model for 2D problems involving infinitely-long plates and three-dimensional (3D) problems applied to circular disks. Islam, Kundu, and Gayen (2019) carried out a theoretical study on the interaction of water-waves with a horizontal rigid disk submerged in the lower layer of a two-layer fluid. In their model, a 2D hypersingular integral equation was developed and was further reduced to a Fredholm integral equation where the unknown function depended only on a single variable. Amplification of the hydrodynamic coefficients was observed as the height of the upper layer decreased. The hypersingular integral equation-based approach was also implemented by, e.g., Porter and Evans (1995), Evans and Porter (1995), for solving water wave interaction with vertical plates.

In the case of a submerged rigid plate/disk made from a porous medium, wave power can be dissipated due to the porosity. Assuming the flow within the porous medium was governed by Darcy's law, Yu and Chwang (1994) examined the wave motion over a submerged porous rigid plate with the 2D boundary-element method (BEM). Moderately large values of the porosity were found to produce the maximum wave power dissipation. In order to study wave interaction with a submerged porous circular disk, Chwang and Wu (1994) developed a 3D theoretical model based on the eigenfunction matching method. In their solution, the wavenumbers, i.e., the eigenvalues of the depth-dependent eigenfunctions, above and below the porous disk were the roots of an identical complex wave dispersion relation. Later, using the Wiener-Hopf method together with the Cauchy integral method, Evans and Peter (2011) gave an analytical solution of the wave scattering for both the semi-infinite porous plate and the finite porous plate backed by a solid vertical wall. A similar method was later employed and developed by Liu and Li (2011) for wave scattering of an offshore submerged porous plate with finite length. In their model, two techniques, i.e., (i) both horizontal and vertical eigenfunction expansions in the regions above and below the plate; and (ii) symmetric and antisymmetric potentials with respect to the vertical axis, were combined, hence finding complex roots was avoided, and simultaneously the size for the system of truncated equations was reduced. Studies regarding the water-wave interaction with two- and multi-layer porous plates can be found in Cho et al. (2013) and Fang, Xiao, and Peng (2017). 
The problem of a submerged impermeable/porous elastic plate has also been investigated by some researchers, but not extensively. Mahmood-Ul-Hassan, Meylan, and Peter (2009) presented the solutions for a submerged impermeable elastic 2D plate with semi-infinite and finite length and a 3D circular disk, respectively, by using the eigenfunction matching method. The wavenumbers of the eigenfunctions above and below the elastic plate were found to be composed of two complex roots, two negative imaginary roots, and an infinite number of positive real roots of a complex wave dispersion relation. The 2D wave scattering problem of a submerged semi-infinite elastic plate was later analytically studied by Williams and Meylan (2012) using the Wiener-Hopf and residue calculus techniques, which had been used by Evans and Peter (2011) for the submerged porous rigid plate. The water-wave interaction with a submerged plate which is not only porous but also flexible is more complicated than those in the case of a submerged plate which is either rigid, impermeable, or both. Cho and Kim (2000) obtained both BEM-based and analytical solutions for the hydroelastic problem of an offshore submerged porous elastic plate. In both models, the complex displacement of the plate was expanded in terms of a set of natural modes. Hence, apart from the wave diffraction problem, the radiation problems regarding these natural modes had to be solved. Behera and Sahoo (2015) solved the 2D waver scattering problem from a submerged porous elastic plate for three cases, (i) a plate in the open sea, (ii) a plate at a finite distance in front of a rigid wall, (iii) a plate moored on a wall. In all cases, the solution was found by an eigenfunction matching method. The wavenumbers of the eigenfunctions above and below the porous elastic plate were observed to all be complex. It was found that the presence of the submerged porous elastic plate caused a significant amount of wave power to be dissipated, while the waves transmitted to the lee side of the plate were dramatically weakened. More recently, Mohapatra, Sahoo, and Guedes Soares (2018) derived the long-wave equations under the shallow water approximation for the $2 \mathrm{D}$ water-wave interaction with a submerged porous elastic plate. It was shown that the wave energy dissipation was significantly dependent upon the mooring stiffness, porous-effect parameter, and position of the submerged plate.

A submerged elastic plate can be used to absorb power from water waves, provided that piezoelectric layers are bonded to both faces of the flexible substrate. The tension variations at the plate-water interface can be converted into a voltage by the piezoceramic layers owing to the piezoelectric effect, and in this way, the elastic motion excited by water waves is transformed into useful electricity. Renzi (2016) derived a hydroelectromechanical dispersion relation and analysed the coupled hydroelectromechanical dynamics of a 2D piezoelectric plate wave energy converter 
(WEC). In this study the effect of the piezoceramic power take-off (PTO) system was modelled as a complex parameter, analogous to the porous-effect parameter for porous rigid/elastic plates (e.g., Mohapatra, Sahoo, and Guedes Soares (2018)). Later, Buriani and Renzi (2017) extended the model to study a flexible piezoelectric WEC moored on a breakwater. The effect of plate size and its submergence on wave power absorption was not examined in their study.

In most of the above studies concerning a submerged plate, the plate was assumed to be either impermeable or rigid. Although the models developed by Renzi (2016), Behera and Sahoo (2015), Mohapatra, Sahoo, and Guedes Soares (2018) et al. can be used to solve the wave scattering problem of submerged porous elastic plate, they cannot be employed for the more common 3D problems, i.e., when the dimension of the plate along the wave front is not much longer than the incident wavelength. For multiple submerged porous elastic plates/disks deployed in an array, the hydrodynamic interaction between them will affect the wave scattering problem significantly unless they are far away from one another. To the best knowledge of the authors, the water-wave interaction with an array of submerged porous elastic disks has not been studied.

In this paper, water-wave interaction of submerged porous elastic disks is investigated in three dimensions using a theoretical model, which is developed based on linear potential flow theory and an eigenfunciton matching method. Performance of a single submerged porous elastic disk, and an array of disks as well, in terms of near-field wave motion, disk deflection, far-field scattering coefficient, and wave power absorption/dissipation is examined with the employment of the theoretical model.

The rest of this paper is organised as follows. $\S-$ II outlines the mathematical model for wave scattering problem. $\S-$ III presents the theoretical solutions of spatial velocity potentials in the fluid domain. The methods for evaluating the scattered far-field amplitude function and wave power absorption/dissipation are given in $\S-I V$. Validation of the present theoretical model is given in $\S-\mathrm{V}$. The validated model is then applied to carry out a multi-parameter study on the performance of a single submerged porous elastic disk, and an array of disks as well, the results of which can be found in $\S-V I$. Finally, conclusions are drawn in $\S-$ VII.

\section{MATHEMATICAL MODEL}

An array of $N$ submerged porous elastic disks of negligible thickness is considered. Cartesian axes are chosen with the mean free-surface coinciding with the $(x, y)$-plane and $z$ measured ver- 

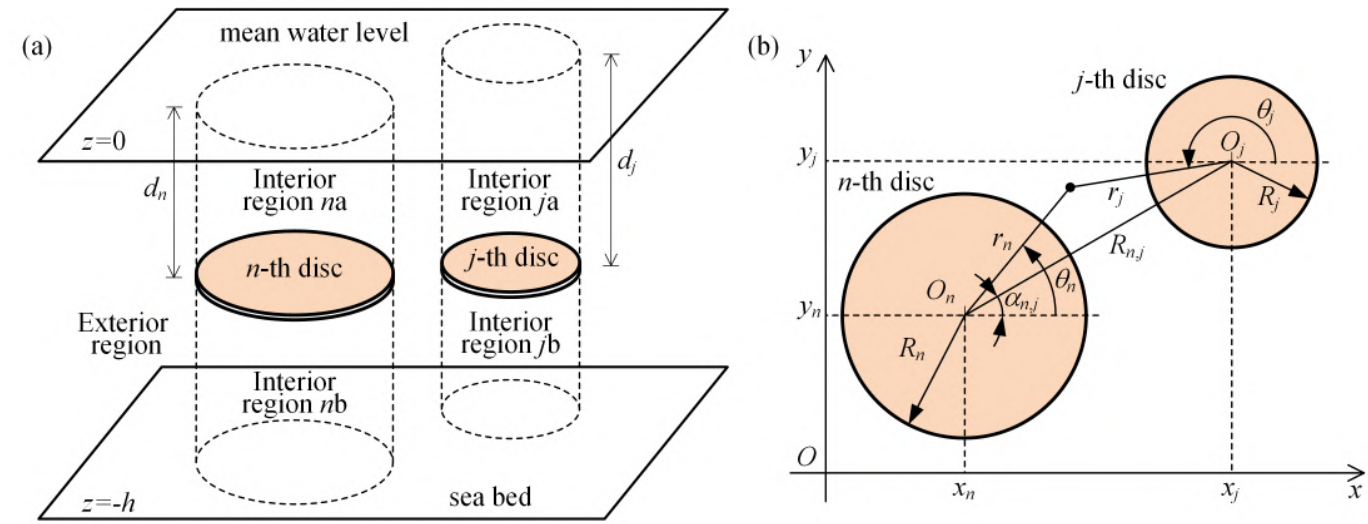

FIG. 1. Schematic of an array of submerged porous elastic disks: (a) side view; (b) plan view.

tically upwards. The fluid bottom is at $z=-h$, and the $n$-th disk with a radius of $R_{n}$ is placed at $z=-d_{n} . N$ local cylindrical coordinate systems $O_{n} r_{n} \theta_{n} z$ for $n=1,2,3, \ldots, N$ are also introduced corresponding to the $n$-th disk. The horizontal position of $O_{n}$ in the Cartesian coordinate system is $\left(x_{n}, y_{n}\right)$. Additionally, one more cylindrical coordinate system $O r_{0} \theta_{0} z$ is defined with its origin coinciding with the Cartesian coordinate system (not plotted in Fig. 1). $R_{n, j}$ and $\alpha_{n, j}$ denote the amplitude and the direction of the vector $\overrightarrow{O_{n} O_{j}}$.

The fluid domain is divided into two types, (I) interior regions, i.e., the regions above and below each disk, which, for the $n$-th disk, are denoted as Interior regions $n$ a and $n \mathrm{~b}$, respectively, and (II) exterior region, i.e., the rest extending towards infinite distance horizontally.

For the array of submerged disks subjected to undisturbed incident waves propagating in the direction $\beta$ relative to the positive $O x$ axis with amplitude $A$ and angular frequency $\omega$, we assume that all amplitudes are small enough such that linear theory applies and we make the usual assumption that the fluid is inviscid, incompressible and irrotational. It is further assumed that all motion is time-harmonic, that the velocity potential in the fluid domain can be denoted as

$$
\Phi(x, y, z, t)=\operatorname{Re}\left[\phi(x, y, z) \mathrm{e}^{-\mathrm{i} \omega t}\right]
$$

where $\phi$ is the time-independent complex spatial velocity potential, and it satisfies the Laplace equation; Re denotes the real part; i denotes the imaginary unit; $t$ is the time.

$\phi$ can be decomposed into $\phi=\phi_{\mathrm{I}}+\phi_{\mathrm{S}}$, where $\phi_{\mathrm{S}}$ is the scattered velocity to be determined, and $\phi_{\mathrm{I}}$ is the well-known velocity potential of undisturbed incident waves that can be expressed as (Zheng and Zhang, 2018; Zheng, Zhang, and Iglesias, 2018)

$$
\phi_{\mathrm{I}}(x, y, z)=-\frac{\mathrm{i} g A}{\omega} Z_{0}(z) \mathrm{e}^{\mathrm{i} k(x \cos \beta+y \sin \beta)},
$$


and

$$
\phi_{\mathrm{I}}\left(r_{n}, \theta_{n}, z\right)=-\frac{\mathrm{i} g A}{\omega} Z_{0}(z) \mathrm{e}^{\mathrm{i} k\left(x_{n} \cos \beta+y_{n} \sin \beta\right)} \sum_{m=-\infty}^{\infty} \mathrm{i}^{m} \mathrm{e}^{-\mathrm{i} m \beta} J_{m}\left(k r_{n}\right) \mathrm{e}^{\mathrm{i} m \theta_{n}},
$$

in the general Cartesian coordinate system $O x y z$ and the local cylindrical coordinate systems $O_{n} r_{n} \theta_{n} z$, respectively, in which $J_{m}$ denotes the Bessel function of the $m$-th order, $g$ is the gravitational acceleration, $k$ denotes the wave number that satisfies $\omega^{2}=g k \tanh (k h)$, and expression of $Z_{0}(z)$ can be found in $\S-$ III.

The displacement of the $n$-th disk about $z=-d_{n}$ can be expressed as

$$
\eta_{0}^{(n)}(x, y, t)=\operatorname{Re}\left[\eta_{n}(x, y) \mathrm{e}^{-\mathrm{i} \omega t}\right]
$$

where $\eta_{n}$ represents the time-independent part of the disk displacement.

The spatial velocity potential, $\phi$, at the interface of the interior regions $n$ a and $n \mathrm{~b}$ can be coupled to the disk displacement function, $\eta_{n}$, in terms of both kinematic and dynamic conditions (Meylan, 2002)

$$
\begin{gathered}
\partial_{z} \phi=-\mathrm{i} \omega \eta_{n}+\mathrm{i} P_{n}\left(\phi_{-}-\phi_{+}\right), \text {on } r_{n} \in\left[0, R_{n}\right], z=-d_{n}, \\
g\left(\chi_{n} \Delta^{2}-\frac{\omega^{2}}{g} \gamma_{n}\right) \eta_{n}+\mathrm{i} \omega\left(\phi_{+}-\phi_{-}\right)=0, \text { on } r_{n} \in\left[0, R_{n}\right], z=-d_{n},
\end{gathered}
$$

where $P_{n}$ could be a complex porosity parameter of the $n$-th disk, the real and imaginary parts of which represent the resistance and inertial effects, respectively. In this paper, for simplification, only the resistance effect is considered, i.e., $P_{n}$ becomes a real porosity parameter. $P_{n}$ may also be interpreted as a PTO damping coefficient, should a particular type of PTO be applied to elastic disks, which work as an array of submerged elastic wave energy converters to absorb wave power rather than to dissipate energy (e.g., Renzi (2016)). + and - denote above and below the disk, respectively; $\chi_{n}=\frac{D_{n}}{\rho g}$ and $\gamma_{n}=\frac{\rho_{n, \mathrm{p}} H_{n}}{\rho}$ denote the flexural rigidity and the mass per unit area of the disk, respectively, scaled with respect to the water density $\rho$, in which $D_{n}$ is the flexual rigidity, $\rho_{n, \mathrm{p}}$ the $n$-th disk density and $H_{n}$ the thickness of the disk. $\Delta$ is the Laplacian operator in the horizontal plane.

Eqs. (5) and (6) can be combined into

$$
\left(\chi_{n} \Delta^{2}-\frac{\omega^{2}}{g} \gamma_{n}\right)\left[\partial_{z} \phi+\mathrm{i} P_{n}\left(\phi_{+}-\phi_{-}\right)\right]+\frac{\omega^{2}}{g}\left(\phi_{+}-\phi_{-}\right)=0 \text {, on } r_{n} \in\left[0, R_{n}\right], z=-d_{n} \text {. }
$$

Apart from the Laplace equation and the boundary condition at the $n$-th disk, the spatial velocity potential should satisfy the following boundary conditions:

$$
\partial_{z} \phi=\frac{\omega^{2}}{g} \phi, \text { on } z=0,
$$




$$
\begin{gathered}
\partial_{z} \phi=0, \text { on } z=-h, \\
\left.\partial_{z} \phi\right|_{z=-d_{n,+}}=\left.\partial_{z} \phi\right|_{z=-d_{n,-}}, \text { on } r_{n} \in\left[0, R_{n}\right] \quad z=-d_{n} .
\end{gathered}
$$

Additionally, in the far-field horizontally, the scattered wave potential $\phi_{\mathrm{S}}$ is subject to the Sommerfeld radiation condition.

The boundary conditions at the edge of each disk should be satisfied as well, which are dependent on the type of disk edge.

For submerged disks having simply supported edges, vanishing of bending moment $F_{M}^{(n)}$ and displacement yields

$$
F_{M}^{(n)}=0 \text { and } \eta_{n}=0 \text {, on } r_{n}=R_{n}
$$

where

$$
F_{M}^{(n)}=\frac{\partial^{2} \eta_{n}}{\partial r_{n}^{2}}+\frac{v}{R_{n}^{2}} \frac{\partial^{2} \eta_{n}}{\partial \theta_{n}^{2}}+\frac{v}{R_{n}} \frac{\partial \eta_{n}}{\partial r_{n}},
$$

in which $v$ denotes Poisson's ratio; $\eta_{n}$ can be expressed in terms of $\phi$ by using Eq. (5).

While, in the case of submerged disks having clamped edges, both displacement and slope vanish at the edge, providing

$$
\eta_{n}=0 \text { and } \frac{\partial \eta_{n}}{\partial r_{n}}=0, \text { on } r_{n}=R_{n}
$$

\section{THEORETICAL SOLUTION TO VELOCITY POTENTIALS}

The velocity potentials in the exterior region and interior regions above and below the $n$-th disk are denoted by $\phi_{0}$ and $\phi_{n}$, respectively. Expressions of them are given as follows.

\section{Exterior region}

The eigenfunction expansion in this region is completely standard and follows from Zheng and Zhang (2018); Zheng, Zhang, and Iglesias (2018)

$$
\phi_{0}=\phi_{\mathrm{I}}+\sum_{n=1}^{N} \sum_{m=-\infty}^{\infty} \sum_{l=0}^{\infty} A_{m, l}^{(n)} H_{m}\left(k_{l} r_{n}\right) Z_{l}(z) \mathrm{e}^{\mathrm{i} m \theta_{n}}
$$

where the accumulative term denotes the scattered wave potential, $\phi_{\mathrm{S}}$, satisfying the Sommerfeld radiation condition; $A_{m, l}^{(n)}$ are the unknown coefficients to be determined; $H_{m}$ is the Hankel function

of the $m$-th order; $Z_{l}(z)=\frac{\cosh \left[k_{l}(z+h)\right]}{\cosh \left(k_{l} h\right)} ; k_{0}=k$ and $k_{l}$ for $l=1,2,3, \ldots$ are the positive real root and the infinite positive imaginary roots of the dispersion relation for the exterior region (Zheng and 
Zhang, 2015, 2016; Zheng, Zhang, and Iglesias, 2019)

$$
\omega^{2}=\left\{\begin{array}{ll}
g k_{l} \tanh \left(k_{l} h\right), & l=0 \\
-g \mathrm{i} k_{l} \tan \left(\mathrm{i} k_{l} h\right), & l=1,2,3, \ldots
\end{array} .\right.
$$

With the employment of Graf's addition theorem for Bessel functions (Abramowitz and Stegun, 1964; Zheng, Zhang, and Iglesias, 2018; Zheng et al., 2019), Eq. (14) can be rewritten in the cylindrical coordinates $O_{n} r_{n} \theta_{n} z$ as:

$$
\begin{aligned}
& \phi_{0}\left(r_{n}, \theta_{n}, z\right)=\phi_{\mathrm{I}}+\sum_{m=-\infty}^{\infty} \sum_{l=0}^{\infty} A_{m, l}^{(n)} H_{m}\left(k_{l} r_{n}\right) Z_{l}(z) \mathrm{e}^{\mathrm{i} m \theta_{n}} \\
& +\sum_{\substack{j=1, m=-\infty \\
j \neq n}}^{N} \sum_{l=0}^{\infty} A_{m, l}^{(j)} Z_{l}(z) \sum_{m^{\prime}=-\infty}^{\infty}(-1)^{m^{\prime}} H_{m-m^{\prime}}\left(k_{l} R_{n, j}\right) J_{m^{\prime}}\left(k_{l} r_{n}\right) \mathrm{e}^{\mathrm{i}\left(m \alpha_{j, n}-m^{\prime} \alpha_{n, j}\right)} \mathrm{e}^{\mathrm{i} m^{\prime} \theta_{n}}, \text { for } r_{n} \leq R_{n, j} .
\end{aligned}
$$

\section{Interior regions}

In the interior regions $n \mathrm{a}$ and $n \mathrm{~b}$, the potential satisfies

$$
\phi_{n}\left(r_{n}, \theta_{n}, z\right)=\sum_{m=-\infty}^{\infty} \sum_{l=-2}^{\infty} B_{m, l}^{(n)} I_{m}\left(\kappa_{l}^{(n)} r_{n}\right) Y_{l}^{(n)}(z) \mathrm{e}^{\mathrm{i} m \theta_{n}},
$$

where $B_{m, l}^{(n)}$ are the unknown coefficients to be determined; $I_{m}$ is the modified Bessel function of the first kind of the $m$-th order;

$$
Y_{l}^{(n)}(z)= \begin{cases}-\sin \left(\kappa_{l}^{(n)} c_{n}\right)\left[\kappa_{l}^{(n)} h \cos \left(\kappa_{l}^{(n)} z\right)+\frac{\omega^{2}}{g} h \sin \left(\kappa_{l}^{(n)} z\right)\right], & z \in\left[-d_{n}, 0\right) \\ \cos \left[\kappa_{l}^{(n)}(z+h)\right]\left[\kappa_{l}^{(n)} h \sin \left(\kappa_{l}^{(n)} d_{n}\right)+\frac{\omega^{2}}{g} h \cos \left(\kappa_{l}^{(n)} d_{n}\right)\right], & z \in\left(-h,-d_{n}\right]\end{cases}
$$

satisfies the free surface condition and the sea bed conditions, i.e., Eqs. (8) and (9), and also the requirement that the normal derivatives above and below the disk match, i.e., Eq.(10); $c_{n}=h-d_{n}$; $\kappa_{l}^{(n)}$ for $l=-2,-1,0,1,2, \ldots$ are the roots of the dispersion relation for the $n$-th submerged porous elastic disk,

$$
\begin{aligned}
& \left(\chi_{n} \kappa^{4}-\frac{\omega^{2}}{g} \gamma_{n}\right)\left[\kappa \sin \left(\kappa d_{n}\right)+\frac{\omega^{2}}{g} \cos \left(\kappa d_{n}\right)\right] \tan \left(\kappa c_{n}\right)= \\
& -\left[\mathrm{i} P_{n}\left(\chi_{n} \kappa^{4}-\frac{\omega^{2}}{g} \gamma_{n}\right)+\frac{\omega^{2}}{g}\right]\left\{\left[\cos \left(\kappa d_{n}\right)-\frac{\omega^{2}}{g \kappa} \sin \left(\kappa d_{n}\right)\right] \tan \left(\kappa c_{n}\right)+\sin \left(\kappa d_{n}\right)+\frac{\omega^{2}}{g \kappa} \cos \left(\kappa d_{n}\right)\right\},
\end{aligned}
$$


which can be derived by applying Eq. (7) for the interior regions $n$ a and $n b$.

The dispersion equation can be further nondimensionalised as

$$
\begin{aligned}
& f_{n}(\bar{\kappa})=\bar{\kappa}\left(\bar{\chi}_{n} \bar{\kappa}^{4}-\bar{\alpha} \bar{\gamma}_{n}\right)\left[\bar{\kappa} \sin \left(\bar{\kappa} \bar{d}_{n}\right)+\bar{\alpha} \cos \left(\bar{\kappa} \bar{d}_{n}\right)\right] \sin \left(\bar{\kappa} \bar{c}_{n}\right) \\
& +\left[\mathrm{i} \bar{P}_{n}\left(\bar{\chi}_{n} \bar{\kappa}^{4}-\bar{\alpha} \bar{\gamma}_{n}\right)+\bar{\alpha}\right](\bar{\kappa} \sin \bar{\kappa}+\bar{\alpha} \cos \bar{\kappa})=0 .
\end{aligned}
$$

where $\bar{\kappa}=\kappa h, \bar{\chi}_{n}=\chi_{n} / h^{4}, \bar{\gamma}_{n}=\gamma_{n} / h, \bar{d}=d / h, \bar{c}_{n}=c_{n} / h, \bar{P}_{n}=P_{n} h$, and $\bar{\alpha}=h \omega^{2} / g$.

Since the dispersion equation is even in $\bar{\kappa}$, the solutions occur in pairs of plus and minus. With consideration of the properties of Eq. (17) and $I_{m}\left(\kappa_{l}^{(n)} r_{n}\right)$ regarding the sign of $\kappa_{l}^{(n)}$, only the roots that lie in half of the entire complex plane are required to be discussed.

When $\bar{P}=0$, the disk turns into an impermeable submerged elastic disk. For such circumstances, there are two positive imaginary roots, in general, such that $\bar{\kappa}_{l}^{(n)} \in \mathrm{i} \mathbb{R}^{+}$for $l=0,1$, supporting propagating waves, and an infinite number of positive real roots, $\bar{\kappa}_{l}^{(n)} \in \mathbb{R}^{+}$for $l=$ $2,3,4, \ldots$, corresponding to evanescent modes. Additionally, there are two complex roots, $\bar{\kappa}_{-1}^{(n)} \in$

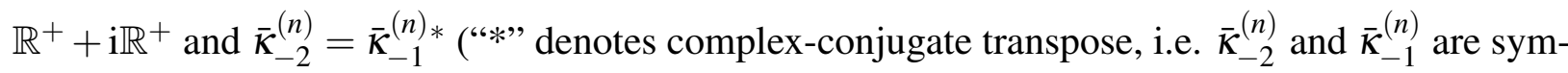
metric in the complex plane with respect to the real axis), which support damped propagating waves. More detailed properties of the dispersion equation for impermeable submerged elastic disk/plate can be found in Mahmood-Ul-Hassan, Meylan, and Peter (2009).

For the case with $\bar{P}=0$, the roots $\bar{\kappa}_{l}^{(n)}$ for $l=0,1,2,3, \ldots$ are evaluated by using a bisection method, and the two complex roots $\bar{\kappa}_{-1}^{(n)}$ and $\bar{\kappa}_{-2}^{(n)}$ can be obtained by means of Muller's method (Press et al., 2007). The roots of the dispersion relation for $\bar{P} \neq 0$ are then derived by using the homotopy method, starting with the corresponding roots for the case of $\bar{P}=0$ (Meylan, Bennetts, and Peter, 2017).

At the interfaces between the exterior region and interior regions, the following continuity conditions of pressure and radial velocity should be satisfied:

(i) Continuity of pressure at the boundary $r_{n}=R_{n}$ :

$$
\left.\phi_{0}\right|_{r_{n}=R_{n}}=\left.\phi_{n}\right|_{r_{n}=R_{n}}, \quad z \in(-h, 0)
$$

(ii) Continuity of radial velocity at the boundary $r_{n}=R_{n}$ :

$$
\left.\frac{\partial \phi_{0}}{\partial r_{n}}\right|_{r_{n}=R_{n}}=\left.\frac{\partial \phi_{n}}{\partial r_{n}}\right|_{r_{n}=R_{n}}, \quad z \in(-h, 0) .
$$


The type-dependent edge conditions, i.e., Eq. (11) or (13), can be appended to the continuity conditions to close the system. A complex linear matrix equation is then derived by using the orthogonality characteristics of $Z_{l}(z)$ and $\mathrm{e}^{\mathrm{i} m \theta_{n}}$, and the eigenfunction-matching method. The unknown coefficients $A_{m, l}^{(n)}$ and $B_{m, l}^{(n)}$ can then be calculated by solving such a complex linear matrix equation. For more information about the derivation and calculation for the unknown coefficients, see A.

Once the unknown coefficients are obtained, the spatial velocity potential all over the fluid domain is known, and the response of the water surface about $z=0, \eta_{0}$ can be calculated in a straightforward manner

$$
\eta_{0}(x, y, t)=\operatorname{Re}\left[\eta(x, y) \mathrm{e}^{-\mathrm{i} \omega t}\right]=\operatorname{Re}\left[\frac{\mathrm{i} \omega}{g} \phi(x, y, 0) \mathrm{e}^{-\mathrm{i} \omega t}\right]
$$

where $\eta$ is the time-independent complex amplitude of the water elevation.

The displacement of the $n$-th disk about $z=-d_{n}$ can be calculated in terms of $B_{m, l}^{(n)}$ as

$$
\begin{aligned}
\eta_{n} & =\frac{1}{\omega}\left[P_{n}\left(\phi_{-}-\phi_{+}\right)+\mathrm{i} \partial_{z} \phi\right] \\
& =\frac{1}{\omega} \sum_{m=-\infty}^{\infty} \sum_{l=-2}^{\infty} B_{m, l}^{(n)} I_{m}\left(\kappa_{l}^{(n)} r_{n}\right)\left[P_{n}\left(\kappa_{l}^{(n)} h \sin \left(\kappa_{l}^{(n)} h\right)+\frac{\omega^{2}}{g} h \cos \left(\kappa_{l}^{(n)} h\right)\right)\right. \\
& \left.-\mathrm{i} \kappa_{l}^{(n)} \sin \left(\kappa_{l}^{(n)} c_{n}\right)\left(\kappa_{l}^{(n)} h \sin \left(\kappa_{l}^{(n)} d_{n}\right)+\frac{\omega^{2}}{g} h \cos \left(\kappa_{l}^{(n)} d_{n}\right)\right)\right] \mathrm{e}^{\mathrm{i} m \theta_{n}} \\
& =\frac{\mathrm{i} \omega h}{g} \sum_{m=-\infty}^{\infty} \sum_{l=-2}^{\infty} \frac{\kappa_{l}^{(n)} \sin \left(\kappa_{l}^{(n)} h\right)+\frac{\omega^{2}}{g} \cos \left(\kappa_{l}^{(n)} h\right)}{\chi_{n}\left(\kappa_{l}^{(n)}\right)^{4}-\frac{\omega^{2}}{g} \gamma_{n}} B_{m, l}^{(n)} I_{m}\left(\kappa_{l}^{(n)} r_{n}\right) \mathrm{e}^{\mathrm{i} m \theta_{n}},
\end{aligned}
$$

where Eqs. (5) and (19) are applied.

\section{FAR-FIELD SCATTERING COEFFICIENT AND WAVE POWER ABSORPTION/DISSIPATION}

\section{A. Far-field scattering coefficient}

In the fluid domain, far away from an array of porous elastic plates, only the propagating modes exist in the scattered waves. With the asymptotic forms of $H_{m}$ for $r_{0} \rightarrow \infty$,

$$
H_{m}\left(k r_{0}\right)=\sqrt{2 / \pi} \mathrm{e}^{-\mathrm{i}(m \pi / 2+\pi / 4)}\left(k r_{0}\right)^{-1 / 2} \mathrm{e}^{\mathrm{i} k r_{0}} \quad \text { for } r_{0} \rightarrow \infty
$$

the scattered wave potential $\phi_{\mathrm{S}}$, i.e., the accumulative term in Eq. (14), can be rewritten as 


$$
\phi_{\mathrm{S}}=\sqrt{2 / \pi} Z_{0}(z) \sum_{n=1}^{N} \sum_{m=-\infty}^{\infty} A_{m, 0}^{(n)} \mathrm{e}^{-\mathrm{i}(m \pi / 2+\pi / 4)}\left(k r_{n}\right)^{-1 / 2} \mathrm{e}^{\mathrm{i} k r_{n}} \mathrm{e}^{\mathrm{i} m \theta_{n}}, \quad r_{0} \rightarrow \infty,
$$

which can be further expressed in the global cylindrical coordinate system $O_{0} r_{0} \theta_{0} z$ as,

$$
\begin{aligned}
\phi_{\mathrm{S}} & =\sqrt{2 / \pi}\left(k r_{0}\right)^{-1 / 2} \mathrm{e}^{\mathrm{i} k r_{0}} Z_{0}(z) \sum_{n=1}^{N} \sum_{m=-\infty}^{\infty} A_{m, 0}^{(n)} \mathrm{e}^{-\mathrm{i} k R_{0, n} \cos \left(\alpha_{0, n}-\theta_{0}\right)} \mathrm{e}^{-\mathrm{i}(m \pi / 2+\pi / 4)} \mathrm{e}^{\mathrm{i} m \theta_{0}} \\
& =A_{S}\left(\theta_{0}\right) \frac{g}{\mathrm{i} \omega} \sqrt{2 \pi}\left(k r_{0}\right)^{-1 / 2} \mathrm{e}^{\mathrm{i}\left(k r_{0}-\pi / 4\right)} Z_{0}(z), \quad r_{0} \rightarrow \infty,
\end{aligned}
$$

where $A_{S}$ is the so-called far-field scattering amplitude that is independent of $r_{0}$ and $z$, and can be expressed as

$$
A_{S}\left(\theta_{0}\right)=\frac{\mathrm{i} \omega}{g \pi} \sum_{n=1}^{N} \sum_{m=-\infty}^{\infty} A_{m, 0}^{(n)} \mathrm{e}^{-\mathrm{i} k R_{0, n} \cos \left(\alpha_{0, n}-\theta_{0}\right)} \mathrm{e}^{\mathrm{i} m\left(\theta_{0}-\pi / 2\right)}
$$

\section{B. Wave power absorption/dissipation}

\section{Direct method}

The energy absorbed/dissipated by the $N$ submerged disks due to porosity, $P_{\text {diss }}$, can be calculated in a straightforward way,

$$
\begin{aligned}
& P_{\text {diss }}=\frac{1}{2 \rho \omega} \sum_{n=1}^{N} P_{n} \iint_{\Omega_{n}}|p|^{2} \mathrm{~d} s=\frac{\rho \omega}{2} \sum_{n=1}^{N} P_{n} \iint_{\Omega_{n}}\left|\phi_{-}-\phi_{+}\right|^{2} \mathrm{~d} s \\
& =\frac{\rho \omega}{2} \sum_{n=1}^{N} P_{n} \iint_{\Omega_{n}}\left|\sum_{m=-\infty}^{\infty} \sum_{l=-2}^{\infty} B_{m, l}^{(n)} I_{m}\left(\kappa_{l}^{(n)} r_{n}\right) \mathrm{e}^{\mathrm{i} m \theta_{n}}\left(\kappa_{l}^{(n)} h \sin \left(\kappa_{l}^{(n)} h\right)+\frac{\omega^{2}}{g} h \cos \left(\kappa_{l}^{(n)} h\right)\right)\right|^{2} \mathrm{~d} s,
\end{aligned}
$$

where $p$ denotes the hydrodynamic pressure loading on the disks, $p=\mathrm{i} \omega \rho\left(\phi_{-}-\phi_{+}\right)$; $\Omega_{n}$ represents the area occupied by the $n$-th disk. It is noted that $P_{\text {diss }}=0$ for $P_{n}=0$ and for $P_{n}=\infty$ as well due to a vanishing hydrodynamic pressure, and that $P_{\text {diss }}>0$ for $0<P_{n}<\infty$. Thus there is a maximum of absorbed/dissipated power when the value of $P_{n}$ is well selected.

The dimensionless quantity of $P_{\text {diss }}$ can be defined by

$$
\eta_{\text {diss }}=k P_{\text {diss }} / P_{\text {in }}
$$

in which $P_{\text {in }}$ is the incoming wave power per unit width of the wave front given by:

$$
P_{\text {in }}=\frac{\rho g A^{2}}{2} \frac{\omega}{2 k}\left(1+\frac{2 k h}{\sinh (2 k h)}\right) \text {. }
$$




\section{Indirect method}

Apart from the straightforward way to calculate the power absorption/dissipation by the array of disks, i.e., Eq. (29), the power absorption/dissipation can also be evaluated based on the spatial potentials in the exterior region:

$$
P_{\text {diss }}=\frac{\rho \omega}{4 \mathrm{i}} \iint_{\Omega_{R}}\left(\phi \frac{\partial \phi^{*}}{\partial r_{0}}-\phi^{*} \frac{\partial \phi}{\partial r_{0}}\right) \mathrm{d} s
$$

where $\Omega_{R}$ represents an envisaged vertical cylindrical control surface with its radius denoted by $r_{0}=R_{0}$, which is large enough to enclose all the disks. The derivation process of Eq. (32) can be found in B; When $r_{0}=R_{0} \rightarrow \infty$, Eq. (32) holds as well with the control surface $\Omega_{R}$ replaced by $\Omega_{\infty}$.

It has been derived that the integral in Eq. (32) for $r_{0} \rightarrow \infty$ can be expressed in terms of Kochin functions, $H_{R}$ (Falnes, 2002),

$$
\iint_{\Omega_{\infty}}\left(\phi \frac{\partial \phi^{*}}{\partial r_{0}}-\phi^{*} \frac{\partial \phi}{\partial r_{0}}\right) \mathrm{d} s=\frac{2 \mathrm{i} A g D(k h)}{\omega k} \operatorname{Re}\left[H_{R}(\beta)\right]-\frac{\mathrm{i} D(k h)}{2 \pi k} \int_{0}^{2 \pi}\left|H_{R}\left(\theta_{0}\right)\right|^{2} \mathrm{~d} \theta_{0},
$$

where $H_{R}$ can be obtained from $A_{m, 0}^{(n)}$ as

$$
H_{R}\left(\theta_{0}\right)=2 \sum_{n=1}^{N} \sum_{m=-\infty}^{\infty} A_{m, 0}^{(n)} \mathrm{e}^{-\mathrm{i} k R_{0, n} \cos \left(\alpha_{0, n}-\theta_{0}\right)}(-\mathrm{i})^{m+1} \mathrm{e}^{\mathrm{i} m \theta_{0}}
$$

and

$$
D(k h)=\left[1+\frac{2 k h}{\sinh (2 k h)}\right] \tanh (k h) .
$$

Therefore, the power absorbed/dissipated by the array of submerged porous elastic disks can be evaluated by using an indirect method based on Kochin functions:

$$
P_{\mathrm{diss}}=\frac{\rho \omega D(k h)}{k}\left(\frac{A g}{2 \omega} \operatorname{Re}\left[H_{R}(\beta)\right]-\frac{1}{8 \pi} \int_{0}^{2 \pi}\left|H_{R}\left(\theta_{0}\right)\right|^{2} \mathrm{~d} \theta_{0}\right),
$$

the result of which can be compared with that of the direct method, presenting a way to check the accuracy of the proposed theoretical model.

The straightforward method, i.e., Eq. (29), includes the surface integrals over all the disks with wave modes, including propagating, damped, and evanescent waves, all considered. As a comparison, the indirect method as given in Eq. (36) is composed of only one angular integral regardless of the number of disks, and it produces an accurate evaluation of the wave power absorption/dissipation by merely taking the propagating waves into account, making it more promising. 
TABLE I. Numerical values of the complex eigenvalues $\bar{\kappa}$, i.e., solutions of the dispersion relation for the interior regions, for $\bar{\chi}=0.01, \bar{\gamma}=0.01, \bar{d}=0.2$ and $\bar{\alpha}=2.0$.

\begin{tabular}{ccc}
\hline \hline Eigenvalue & $\bar{P}=0$ & $\bar{P}=1.0$ \\
\hline $\bar{\kappa}_{-2}$ & $2.5820-0.9549 \mathrm{i}$ & $2.8663-0.8175 \mathrm{i}$ \\
$\bar{\kappa}_{-1}$ & $2.5820+0.9549 \mathrm{i}$ & $2.4050+0.9584 \mathrm{i}$ \\
$\bar{\kappa}_{0}$ & $1.9658 \mathrm{i}$ & $-0.0052+1.9660 \mathrm{i}$ \\
$\bar{\kappa}_{1}$ & $3.9996 \mathrm{i}$ & $-0.5145+3.8892 \mathrm{i}$ \\
$\bar{\kappa}_{2}$ & 3.5361 & $3.4875-0.5321 \mathrm{i}$ \\
$\bar{\kappa}_{3}$ & 7.8456 & $7.8542-0.1616 \mathrm{i}$ \\
$\bar{\kappa}_{4}$ & 11.7799 & $11.7936-0.1053 \mathrm{i}$ \\
$\bar{\kappa}_{5}$ & 15.0459 & $15.0905-0.3663 \mathrm{i}$ \\
$\bar{\kappa}_{6}$ & 15.7077 & $15.6721-0.0646 \mathrm{i}$ \\
$\bar{\kappa}_{7}$ & 19.6349 & $19.6324-0.0637 \mathrm{i}$ \\
$\bar{\kappa}_{8}$ & 23.5619 & $23.5622-0.0531 \mathrm{i}$ \\
$\bar{\kappa}_{9}$ & 27.4889 & $27.4909-0.0454 \mathrm{i}$ \\
$\bar{\kappa}_{10}$ & 31.0947 & $31.1135-0.1704 \mathrm{i}$ \\
\hline \hline
\end{tabular}

Although the present theoretical model can be applied to solve the wave scattering problem from multiple submerged disks with different physical and scale parameters, the following sections are focused on identical disks; hence $R_{n}=R, d_{n}=d, P_{n}=P, \chi_{n}=\chi$ and $\gamma_{n}=\gamma$ are employed from now on for simplification.

\section{VALIDATION}

Table I lists the first 13 roots of the dispersion relation of the interior regions calculated by the present model for the submerged impermeable/porous elastic plates with $\bar{P}=0$ and $\bar{P}=1.0$, and the other parameters $\bar{\chi}=0.01, \bar{\gamma}=0.01, \bar{d}=0.2$ and $\bar{\alpha}=2.0$. These roots are also plotted in Figs. 2 and 3, where the contours of $\operatorname{Re}\{f\}=0$ and $\operatorname{Im}\{f\}=0$ are illustrated as well. The roots predicted by the model are found to locate at the intersection points of the contour lines $\operatorname{Re}\{f\}=0$ and $\operatorname{Im}\{f\}=0$ exactly.

As $N=1, \bar{P}=0$ and $\bar{\chi}$ turns infinite, the case becomes wave scattering by a submerged imper- 


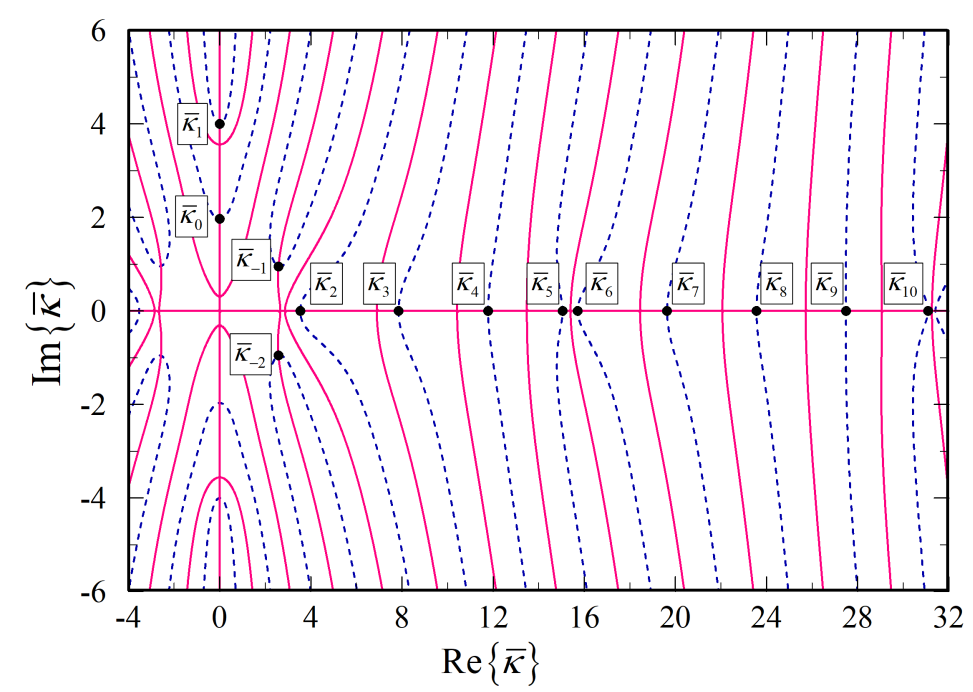

FIG. 2. Location of the first $L+3$ eigenvalues of the dispersion relation $f(\bar{\kappa})=0$ in the complex $\bar{\kappa}$ plane for $L=10$. The dashed blue lines identify the contours $\operatorname{Re}\{f\}=0$, whereas the solid pink lines correspond to the contours $\operatorname{Im}\{f\}=0 .[\bar{\chi}=0.01, \bar{\gamma}=0.01, \bar{d}=0.2, \bar{P}=0, \bar{\alpha}=2.0]$.

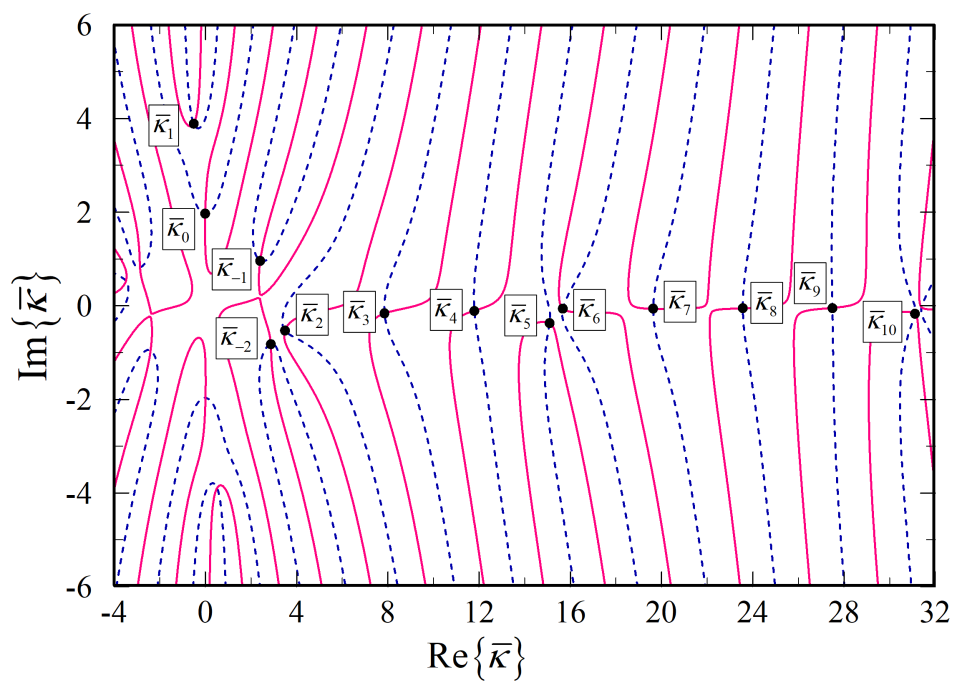

FIG. 3. As figure 2 but for $\bar{P}=1.0$.

meable rigid disk, which was investigated by Yu and Chwang (1993). Fig. 4 illustrates the contour of the dimensionless wave amplitude above the disk. In addition to the wave amplitude contour distribution, the wave amplitudes at three specified positions $\mathrm{P} 1, \mathrm{P} 2$ and $\mathrm{P} 3$, i.e., $(r=R, \theta=0)$, ( $r=R, \theta=\pi / 2)$ and $(r=R, \theta=\pi)$, for the disk with different values of $R$ are examined. Variation of the responses of the dimensionless wave amplitudes at these three points with $R / \lambda$ ( $\lambda$ denotes wavelength) is plotted in Fig. 5, where the results with $\bar{P} \neq 0$ denoting the scattering problem of a 

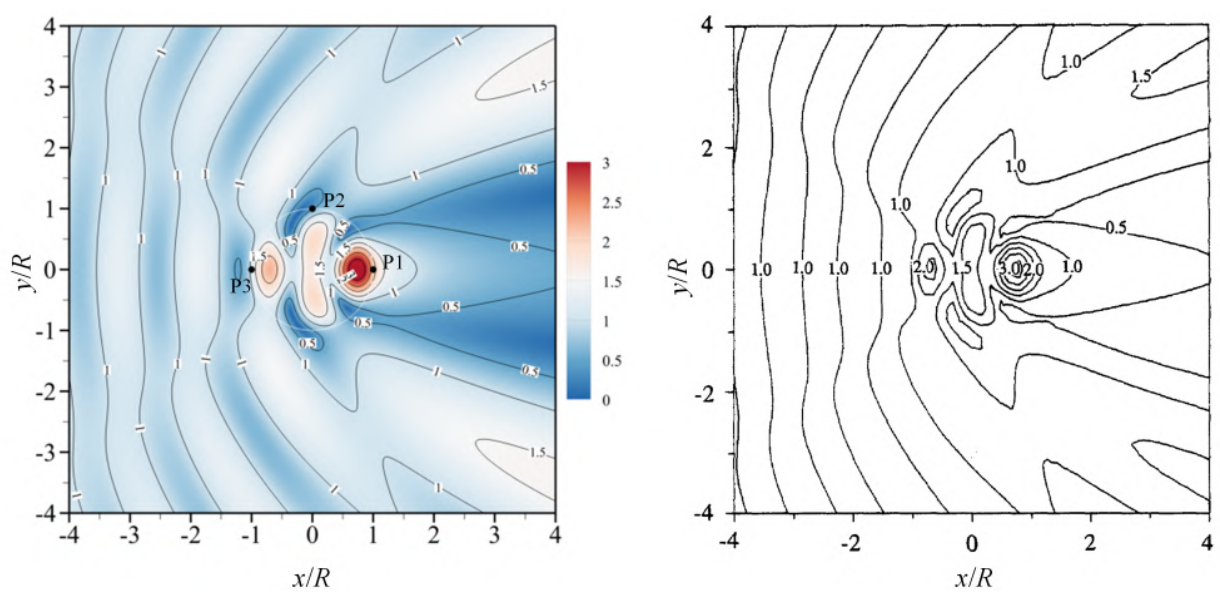

FIG. 4. Dimensionless wave amplitude distribution about a submerged rigid impermeable disk: (left) present results; (right) Yu and Chwang (1993). $\left[N=1, x_{1} / h=y_{1} / h=0, R_{1} / h=1.6, \lambda / h=4.0, \bar{\chi}=10^{3}\right.$, $\bar{\gamma}=0.01, \bar{d}=0.2, \beta=0]$.
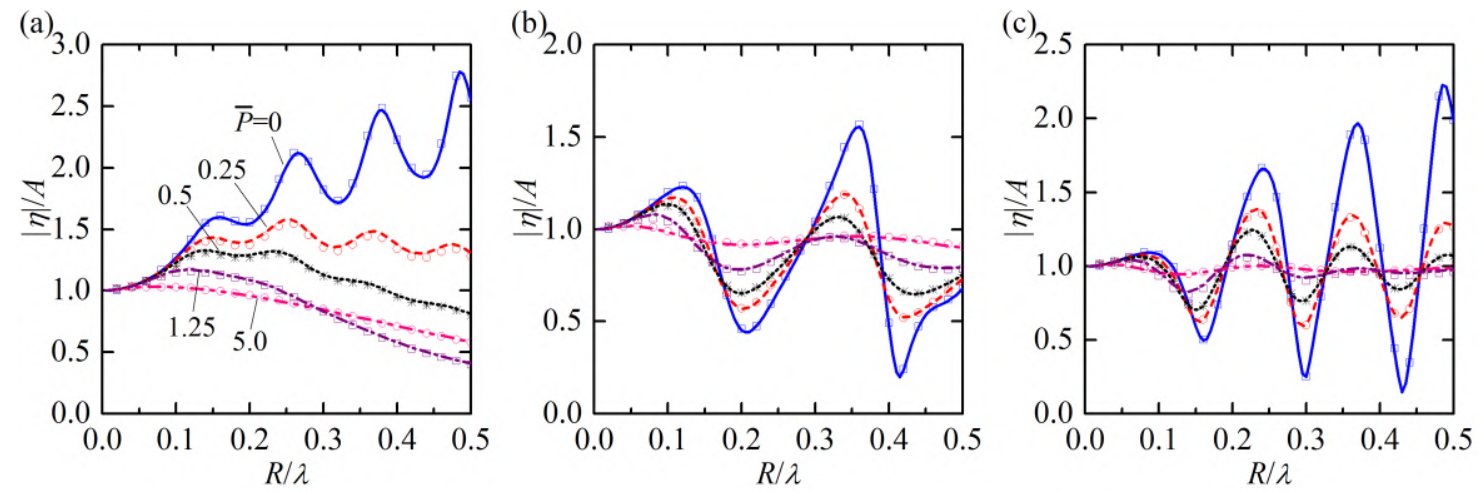

FIG. 5. Variation of dimensionless wave amplitudes at three points above the disk versus disk radius in terms of $R / \lambda$ : (a) P1; (b) P2; (c) P3. [Lines: present results; symbols: Yu and Chwang (1993); Chwang and $\left.\mathrm{Wu}(1994) . N=1, x_{1} / h=y_{1} / h=0, \lambda / h=4.0, \bar{\chi}=10^{3}, \bar{\gamma}=0.01, \bar{d}=0.2, \beta=0\right]$.

submerged porous disk (Chwang and $\mathrm{Wu}, 1994)$ are also included. The present results agree very well with the published data.

Fig. 6 presents the wave power absorption/dissipation by a pair of submerged porous elastic disks, which is calculated by both the direct- and indirect methods. An excellent agreement between them is obtained, which, together with the results plotted in Figs. 2-5, gives confidence in the present model for solving wave scattering and predicting wave motion and wave power absorption/dissipation by submerged porous elastic disks. 

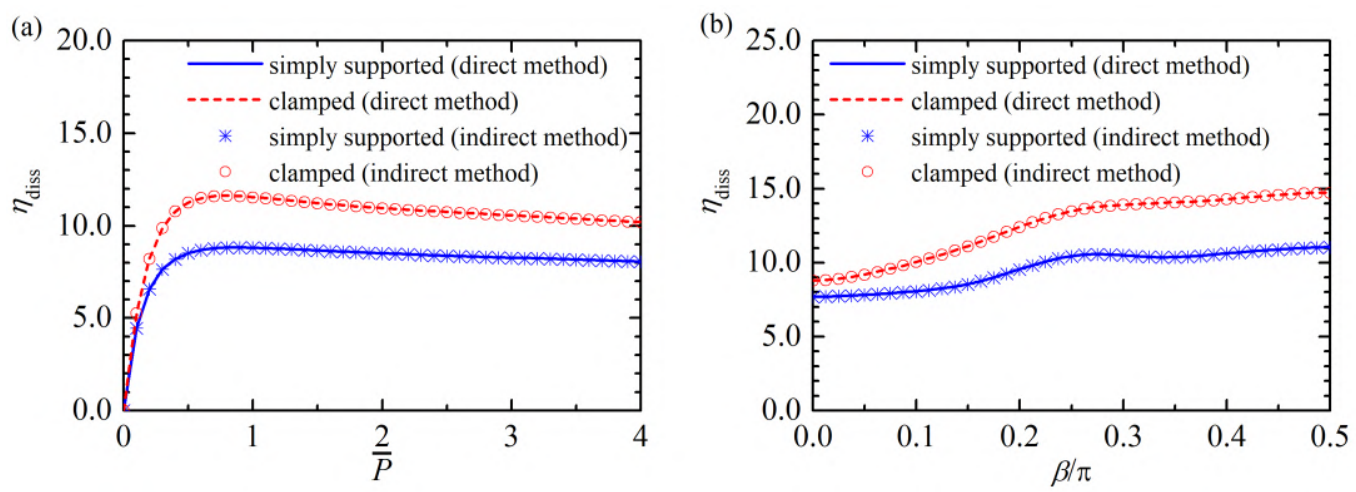

FIG. 6. Wave power absorption/dissipation of a pair of submerged disks with different kinds of edge conditions evaluated by using direct- and indirect methods: (a) variation of $\eta_{\text {diss }}$ with $\bar{P}$ for $\beta=\pi / 6$; (b) variation of $\eta_{\text {diss }}$ with $\beta$ for $\bar{P}=1.0$. [ $N=2,-x_{1} / h=x_{2} / h=2.5, y_{1}=y_{2}=0, R / h=2.0, \bar{\chi}=\bar{\gamma}=0.01, \bar{d}=0.2$, $\bar{\alpha}=2.0]$.

\section{RESULTS AND DISCUSSION}

\section{A. Single submerged porous elastic disk}

First, the performance of a single submerged porous elastic disk is investigated.

\section{Effect of porosity and radius of the disk}

Fig. 7 presents the variation of wave power absorption/dissipation of a single submerged disk with porosity for different radii, and $\bar{\alpha}=2.0, \bar{\chi}=\bar{\gamma}=0.01, \bar{d}=0.2$. The wave power absorption/dissipation is expressed in terms of $4 h^{2} \eta_{\text {diss }} / R^{2}$, which represents the averaged wave power that can be absorbed/dissipated per unit area of the disk.

For the case with $R / h=1.0$, as expected, as $\bar{P}$ increases from 0 towards $4.0,4 h^{2} \eta_{\text {diss }} / R^{2}$ first increases and then decreases after reaching the peak value around $\bar{P}=1.0$ regardless of the edge conditions. A similar trend is observed for the other cases with larger values of $R / h$, while the corresponding $\bar{P}$ where the peak of $4 h^{2} \eta_{\text {diss }} / R^{2}$ occurs is smaller than 1.0, and the declining trend of the curve after reaching its peak becomes much weaker. For most of the computed range of $\bar{P}$, more wave power can be absorbed/dissipated per unit area of the disk with a smaller value of $R / h$.

In Fig. 8, wave power absorption/dissipation of the submerged porous elastic disk in terms of $4 h^{2} \eta_{\text {diss }} / R^{2}$ is plotted against $\bar{\alpha}$ for $\bar{P}=1.0$ and different values of $R / h$. When the wave 

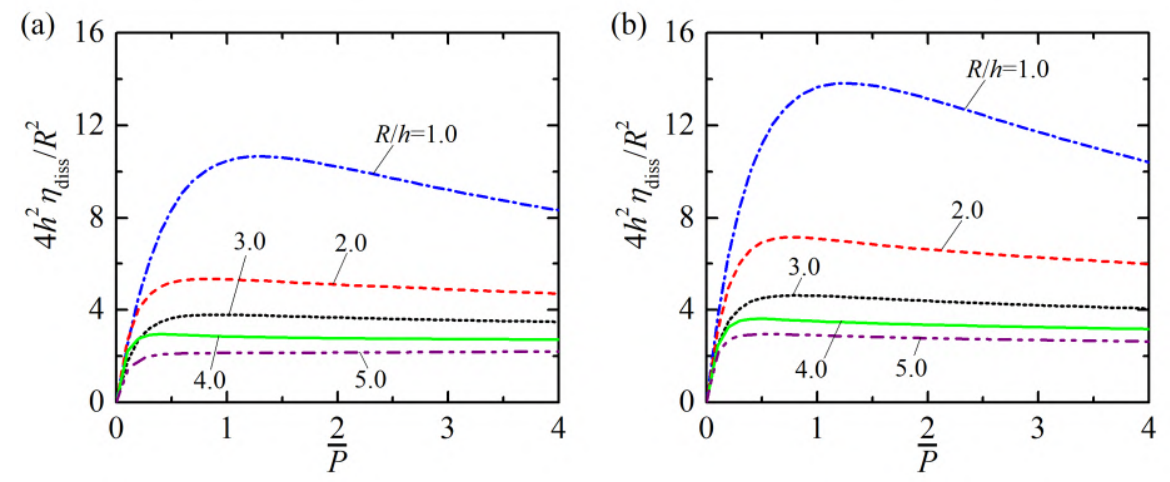

FIG. 7. Variation of wave power absorption/dissipation of a single submerged disk in terms of $4 h^{2} \eta_{\text {diss }} / R^{2}$ with different porosity for different radii: (a) simply supported; (b) clamped. $[N=1, \bar{\alpha}=2.0, \bar{\chi}=\bar{\gamma}=0.01$, $\bar{d}=0.2 . M=30$ and $L=20$ are adopted to obtain the converged results].
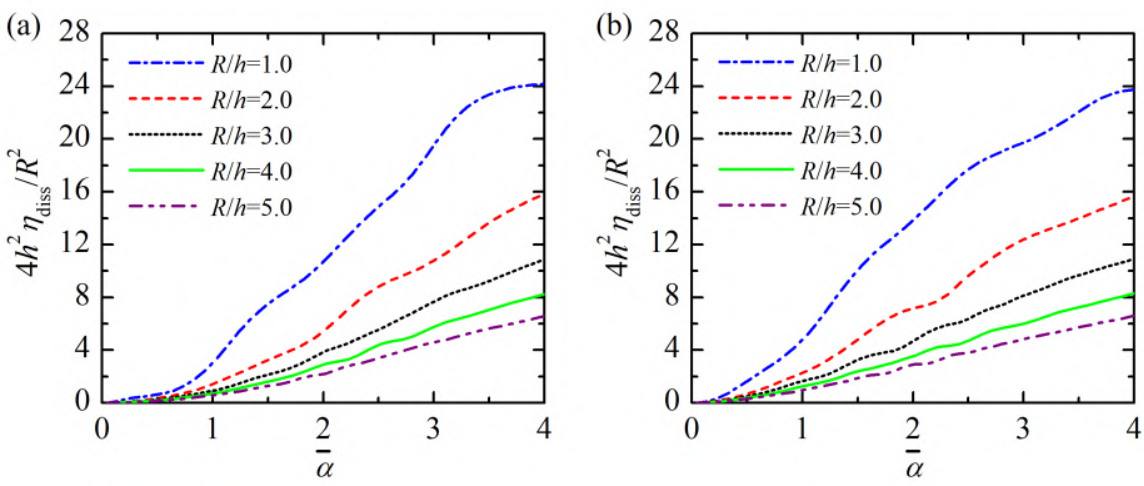

FIG. 8. Frequency response of wave power absorption in terms of $4 h^{2} \eta_{\text {diss }} / R^{2}$ for a submerged porous elastic disk with different radii: (a) simply supported; (b) clamped. $[N=1, \bar{P}=1.0, \bar{\chi}=\bar{\gamma}=0.01, \bar{d}=0.2$. $M=30$ and $L=20$ are adopted to obtain the converged results].

length is extremely large, i.e., $\bar{\alpha}$ and $k \rightarrow 0$, we have $4 h^{2} \eta_{\text {diss }} / R^{2} \rightarrow 0$. In the computed range of wave conditions, i.e., $\bar{\alpha} \in(0,4.0], 4 h^{2} \eta_{\text {diss }} / R^{2}$ increases monotonically with the increasing of $\bar{\alpha}$ regardless of the disk radius and its edge conditions. For long waves, e.g., $\bar{\alpha}<2.0$, the disk with its edge clamped shows a better performance in wave power absorption/dissipation compared to that with a simply supported edge. However, for short waves, the advantage of the clamped edge is not obvious, and for some particular circumstances, e.g., $R / h=1.0$ for $\bar{\alpha} \in(3.0,4.0]$, a simply supported edge condition is even more conducive to wave power absorption/dissipation.

The contour of the near-field wave motion above a submerged impermeable/porous elastic disk with a simply supported edge condition for different radii and also the corresponding disk motion 

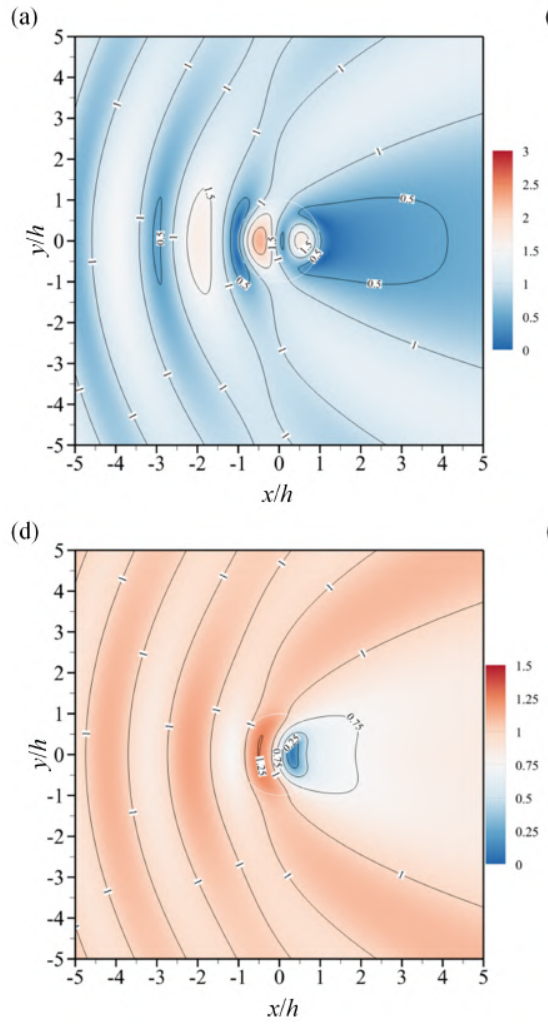

(b)
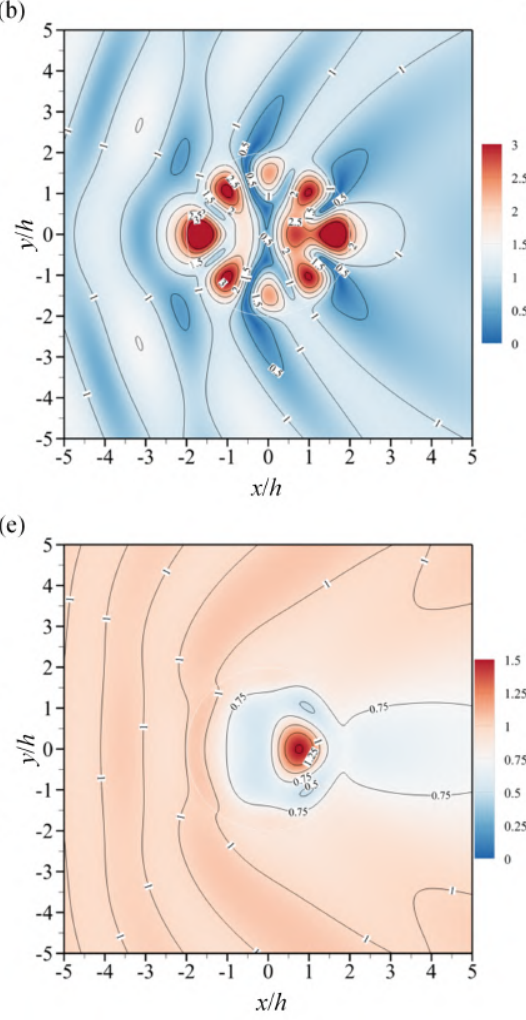

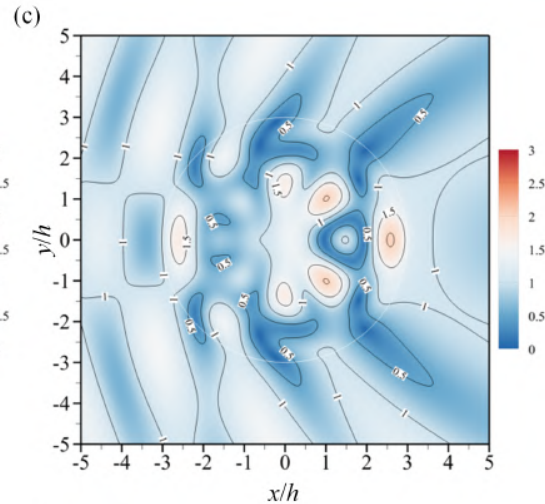

(f)

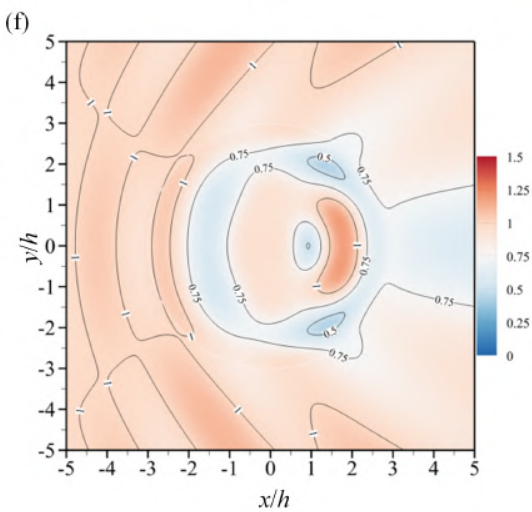

FIG. 9. Contour of the wave amplitude above a single submerged porous elastic disk $|\eta| / A$ with different values of porosity parameter and radius: (a) $\bar{P}=0, R / h=1.0$; (b) $\bar{P}=0, R / h=2.0$; (c) $\bar{P}=0, R / h=3.0$; (d) $\bar{P}=1.0, R / h=1.0$; (e) $\bar{P}=1.0, R / h=2.0$; (f) $\bar{P}=1.0, R / h=3.0 ;[N=1, \bar{\alpha}=2.0, \beta=0, \bar{\chi}=\bar{\gamma}=0.01$, $\bar{d}=0.2$, simply supported]

are plotted in Figs. 9 and 10.

For the impermeable disk with $R / h=1.0$, there are two peaks of $|\eta| / A$ occurring right above the disk (Fig. 9a), in which the larger one with the peak value $|\eta| / A=2.26$ is located at the windward side. There are two peaks of the disk motion $\left|\eta_{1}\right| / A$ as well, whereas the larger peak with the peak value $\left|\eta_{1}\right| / A=1.92$ occurs at the leeward side (Fig. 10a). At the leeward side of the disk in the plotted domain, there is a large area where the surface waves are dramatically attenuated $(|\eta| / A<0.5)$. The regions of $|\eta| / A<0.5$ are also found at the windward side of the disk, and at the area between the two peaks of $|\eta| / A$ right above the disk as well but with a very small area.

For the impermeable disk with $R / h=2.0$, nine peaks of $|\eta| / A$ are observed in the interior region, in which eight are distributed along the edge of the disk, and the remaining one is located between them (Fig. 9b). The most significant value of $|\eta| / A$ can be as large as 3.94, demonstrating dramatic wave focusing effect of the submerged impermeable elastic disk. Meanwhile, there are 

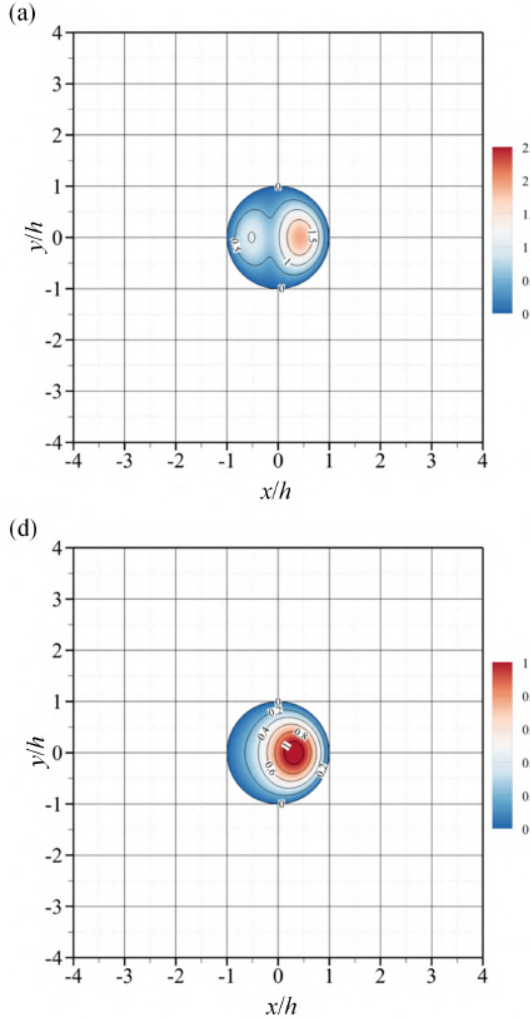

(b)
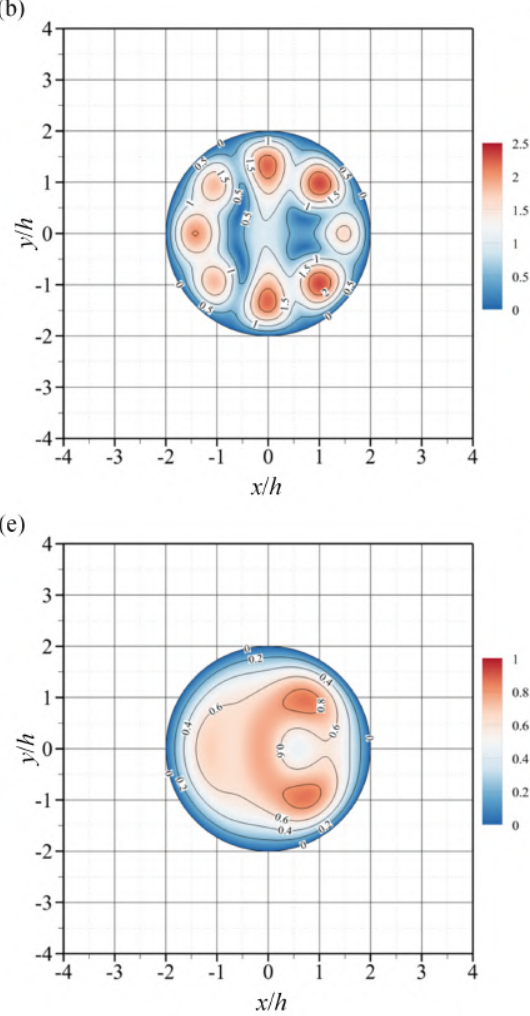

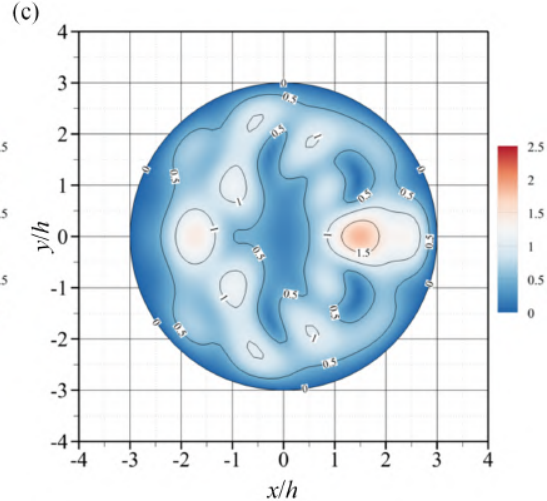

(f)

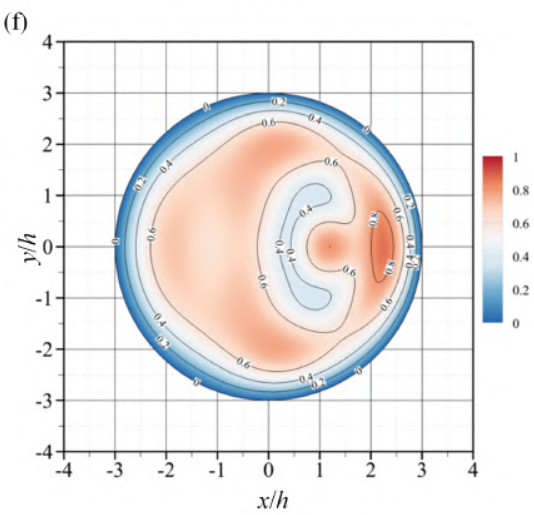

FIG. 10. Motion amplitude of the single submerged porous elastic disk $\left|\eta_{1}\right| / A$ with different values of porosity parameter and radius: (a) $\bar{P}=0, R / h=1.0$; (b) $\bar{P}=0, R / h=2.0$; (c) $\bar{P}=0, R / h=3.0$; (d) $\bar{P}=1.0, R / h=1.0$; (e) $\bar{P}=1.0, R / h=2.0$; (f) $\bar{P}=1.0, R / h=3.0 ;[N=1, \bar{\alpha}=2.0, \beta=0, \bar{\chi}=\bar{\gamma}=0.01$, $\bar{d}=0.2$, simply supported]

some wave attenuation areas between the wave focusing areas as well extending outwards with $|\eta| / A<0.5$. It is noted that at the leeward side of the disk, there is an area of the exterior region that the incident waves are amplified rather than attenuated. As shown in Fig. 10b, there are eight peaks of the disk deflection, and the largest peak value of $\left|\eta_{1}\right| / A$ is 2.33 .

When $R / h=3.0$, the incident waves are attenuated at the majority of the region right above the disk (Fig. 9c). At the very windward and leeward sides, and also at the central part of the interior region, the incident waves are amplified and the largest value of $\left|\eta_{1}\right| / A$ is 2.05 . The corresponding contour of the disk deflection as plotted in Fig. 10c shows that the maximum deflection $\left|\eta_{1}\right| / A=1.85$ can be obtained at $(x / h, y / h)=(1.5,0)$.

As the submerged elastic disk changes from impermeable $(\bar{P}=0)$ into porous $(\bar{P}=1.0)$, the effect of the disk on the overall near-field wave motion is found to be clearly weakened. So is the disk deflection, as expected. Moreover, the contour shapes of both the surface wave motion 
and disk motion change significantly. Taking the case of $R / h=2.0$ as an example (Figs. $9 \mathrm{~b}$ and 9e): as $\bar{P}$ increases from 0 to 1.0 , the multiple peaks of $|\eta| / A$ disappear, and only one single peak remains with the peak value $|\eta| / A=1.52$; at the leeward side of the disk in the exterior region, the previous wave amplification area becomes a wave attenuation area; the eight peaks of $\left|\eta_{1}\right| / A$ merge into three with the largest peak value 0.87 .

In addition to the near-field wave motion and the corresponding disk motion of the submerged impermeable/porous elastic disk, the far-field scattering coefficients of a single submerged elastic disk with different values of the porosity parameter $(\bar{P}=0$ and 1.0$)$ and radius $(R / h=1.0,2.0$ and 3.0) for $\bar{\alpha}=2.0$ and $\beta=0$ are also examined (see Fig. 11). The shape of the far-field scattering coefficient curve is found to be strongly dependent on $R / h$. The larger the disk radius, the stronger the fluctuations of the far-field scattering coefficient curve in terms of both the oscillating amplitude and frequency. Additionally, the position where the largest peak value of the far-field scattering coefficient occurs is also affected by $R / h$. For the impermeable disk with the simply supported edge condition, the largest values of $\left|A_{\mathrm{S}}\right| / A$ are observed at $\theta_{0}= \pm \pi, \pm 0.83 \pi$, and 0 , for $R / h=$ 1.0, 2.0 and 3.0, respectively. The peaks in wave reflection (Fig. 11) may be due to wave trapping by the porous disk (Sahoo, Lee, and Chwang, 2000). The permeability of the disk obviously affects the phenomenon of wave trapping. Existence of the porosity reduces the overall far-field scattering coefficients, except those for some particular directions, e.g., $\theta_{0} \in(-0.17 \pi, 0.17 \pi)$ for the disk with $R / h=2.0$, simply supported edge condition; and $\theta_{0} \in(-\pi,-0.9 \pi) \cup(0.9 \pi, \pi)$ for the disk with $R / h=3.0$, clamped edge condition. For a porous elastic disk, there is a flow passing through the disk that may weaken the process of wave refraction and reflection, leading to the dissipation/absorption of the incident wave power. In the limit, as $P_{n}$ tends to infinity, there would be no effect of the disk at all on wave scattering.

\section{Effect of submergence of the disk}

Apart from the radius of the disk, the submergence of the disk is another significant parameter affecting wave scattering by a submerged porous elastic disk.

Fig. 12 plots the wave power absorption/dissipation of a single submerged disk against $\bar{P}$ for different values of $d / h$. It is observed that, for $\bar{P}>1.3$, the wave power absorbed/dissipated by the porous elastic disk decreases with the increase of the disk submergence. This is reasonable since the fluid motion becomes weaker and weaker with the increase of submergence, and indeed most 

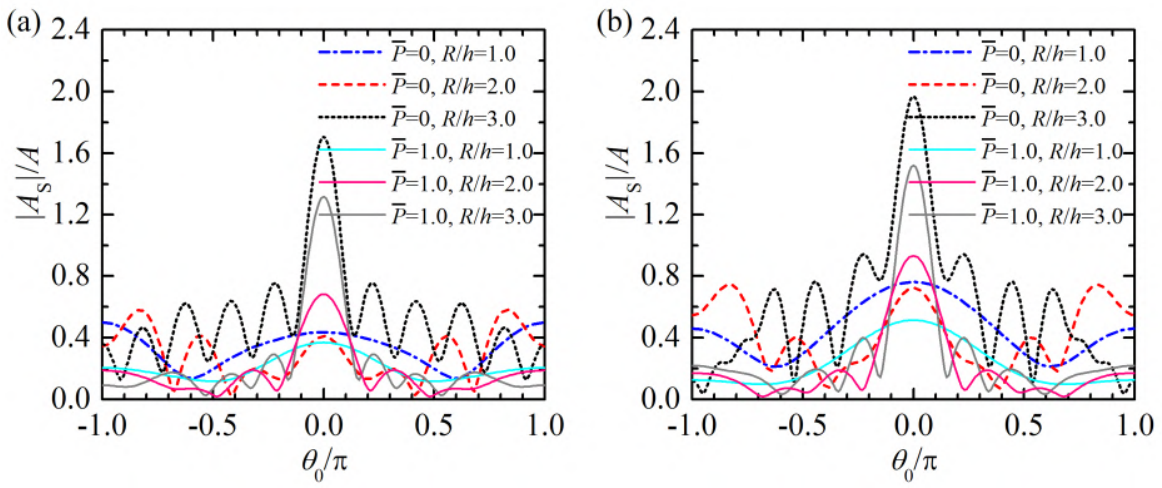

FIG. 11. Far-field scattering coefficient of a single submerged porous elastic disk with different values of porosity parameter and radius: (a) simply supported; (b) clamped. $[N=1, \bar{\alpha}=2.0, \beta=0, \bar{\chi}=\bar{\gamma}=0.01$, $\bar{d}=0.2]$.

of the wave power is distributed at no more than a quarter wavelength below the seawater level. Interestingly, this appears to be not the case when $\bar{P}<1$.2. In the range of $\bar{P} \in(0.3,1.2)$, the disk with $d / h=0.15$ is found to capture more wave power than the other cases examined, including the case with a smaller submergence, i.e., $d / h=0.1$. This might be explained from the point of view of wave resonance. Similar to wave power extraction from a traditional heave wave energy converter, e.g., Pages 51-52 (Falnes, 2002), the wave power absorbed/dissipated by an elastic disk depends not only on the wave excitation force/pressure and porosity parameter but also on the resonance condition. Compared to the case of $d / h=0.1$, the wave resonance is more likely to be excited by the case of $d / h=0.15$. For $\bar{P} \in(0.3,1.2)$, the resonance condition plays a more important role than that of the wave excitation force/pressure in affecting wave power absorption/dissipation, resulting in a larger $\eta_{\text {diss }}$ in spite of a larger submergence. This is in line with the practical requirement that, the disk should remain continuously submerged and its submergence cannot be very shallow, especially for wave conditions with large amplitude or large tidal range, or both.

The corresponding near-field wave motion above the submerged porous elastic disk with different submergence values $d / h=0.1,0.15,0.2,0.25$ and 0.3 for $\bar{P}=1.0$, and the disk deflection, are given in Figs. 13 and 14, respectively. The disturbance induced by the disk on the wave motion at the exterior region is weakened with the increase of the disk submergence. Although the wave scattering is reduced as well for the majority part of the interior region, the maximum magnification of the wave amplitude increases first and then decreases, rather than decreasing monotonically with the increase of the disk submergence $d / h$ from 0.1 to 0.25 . A similar trend is observed to the 
(a)

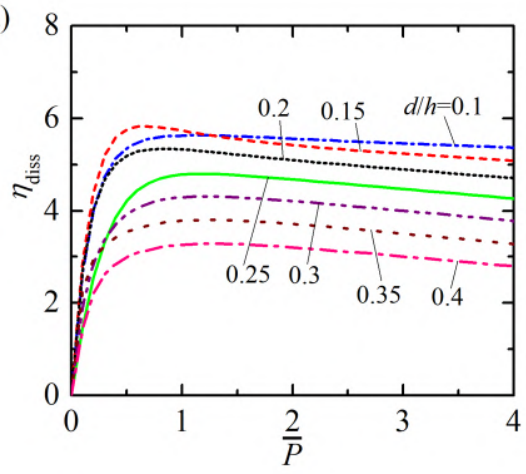

(b)

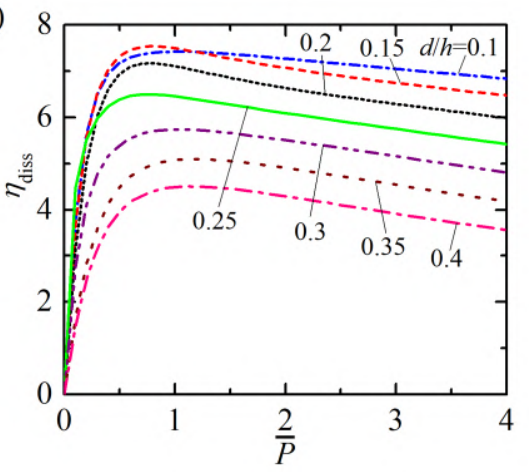

FIG. 12. Variation of wave power absorption/dissipation of a single submerged disk with different porosity for different submergence: (a) simply supported; (b) clamped. $[N=1, \bar{\alpha}=2.0, R / h=2.0, \bar{\chi}=\bar{\gamma}=0.01]$.

disk deflection via disk submergence. More specifically, the peak values of the wave amplitude and disk deformation are $\left(|\eta| / A,\left|\eta_{1}\right| / A\right)=(1.45,0.83),(1.65,0.87),(1.52,0.87)$ and $(1.35,0.83)$, respectively, for $d / h=0.1,0.15,0.2$ and 0.25 .

Fig. 15 illustrates the variation of the far-field scattering coefficient against direction for a submerged porous elastic disk with different submergence. The far-field scattering coefficient at the very leeward side of the disk, e.g., $\theta_{0} \in(-0.15 \pi, 0.15 \pi)$, is much larger compared to those of the remaining directions. The far-field scattering coefficient at the leeward side is found to decrease monotonously with the increase of disk submergence. For the remaining directions, there is no clear changing rule of $\left|A_{\mathrm{S}}\right| / A$ versus $d / h$.

\section{B. Multiple submerged porous elastic disks}

In this subsection, the performance of an array of identical submerged porous elastic disks is investigated.

\section{Effect of the distance between the disk centers}

When the disks in an array are deployed far away from one another, the hydrodynamic interaction between them can be neglected, and each disk performs like a single disk in isolation. However, a more common circumstance is that the disks are arranged not far away but close to one another. In such a case, the hydrodynamic interaction between them may play a significant role in affecting the wave scattering problem. 

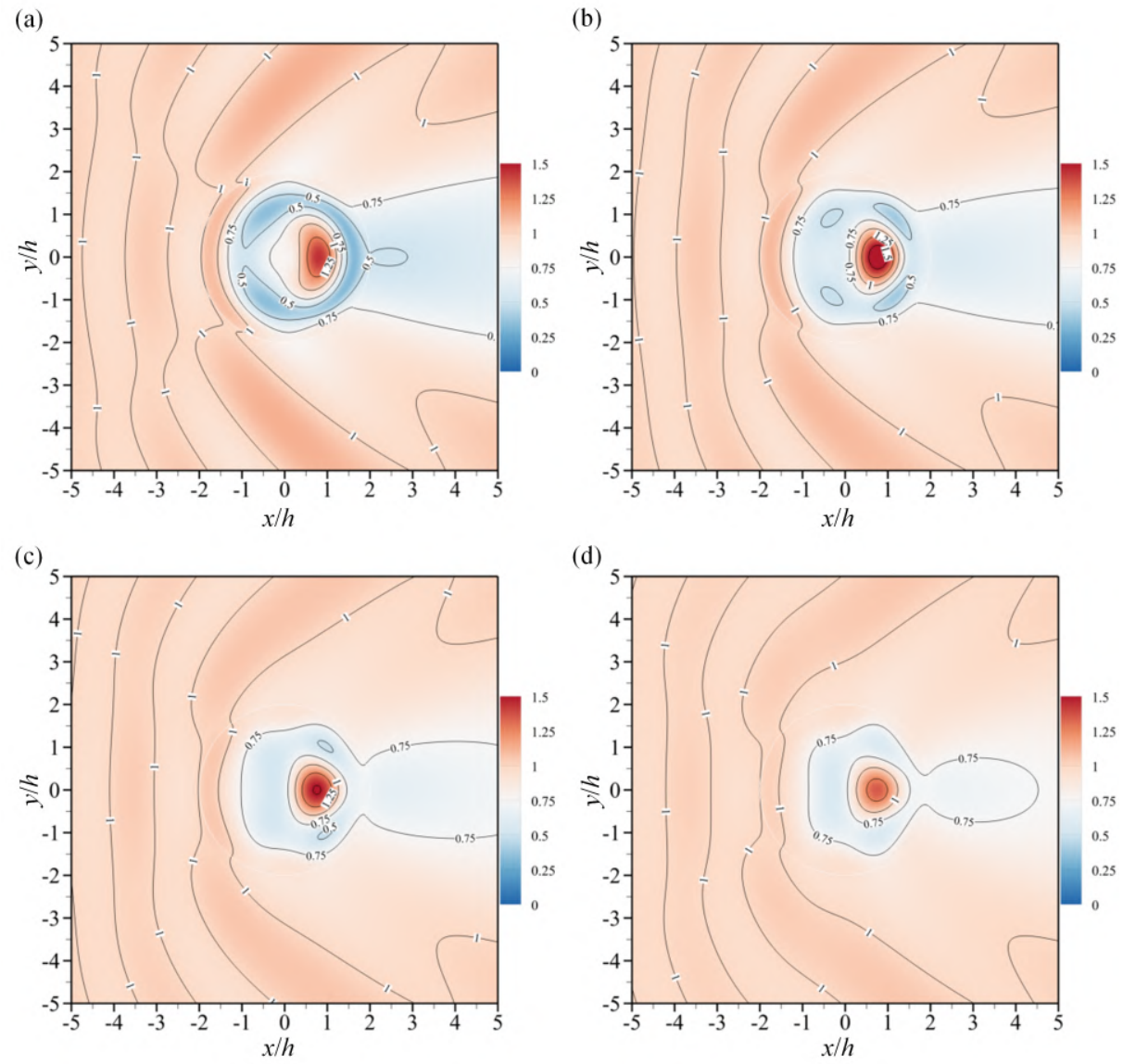

FIG. 13. Contour of the wave amplitude above a single submerged porous elastic disk $|\eta| / A$ with different submergence: (a) $d / h=0.1$; (b) $d / h=0.15$; (c) $d / h=0.2$; (d) $d / h=0.25$. [N=1, $\bar{\alpha}=2.0, \beta=0$, $\bar{\chi}=\bar{\gamma}=0.01, R / h=2.0, \bar{P}=1.0$, simply supported $]$

Fig. 16 presents the contour of wave power absorption/dissipation versus the distance between the centres of a pair of submerged porous elastic disks and the porosity parameter of the disks. It is indicated that, for any specified distance between the two disks, the wave power absorption/dissipation reaches the maximum around $\bar{P}=1.0$ regardless of the edge conditions. For any certain value of $\bar{P} \in(0.5,4]$, as $R_{1,2} / h$ increases from 5.0 to $8.0, \eta_{\text {diss }}$ first increases and then decreases after reaching the maximum. In the computed range of $\bar{P}$ and $R_{1,2} / h$, the peak values of $\eta_{\text {diss }}$ are 11.1 and 14.9 for the simply supported and clamped edge conditions, respectively, occurring at $\left(\bar{P}, R_{1,2} / h\right)=(0.9,5.26)$ and $(0.75,5.38)$, separately.

The near-field wave motion above the pair of submerged porous elastic disks with simply supported edge condition, and the corresponding deflection of the disks with different spacing distance $R_{1,2} / h=5.0,6.0,7.0,8.0, \bar{P}=1.0, \bar{\alpha}=2.0$ and $\beta=0$ are also examined but not plotted 

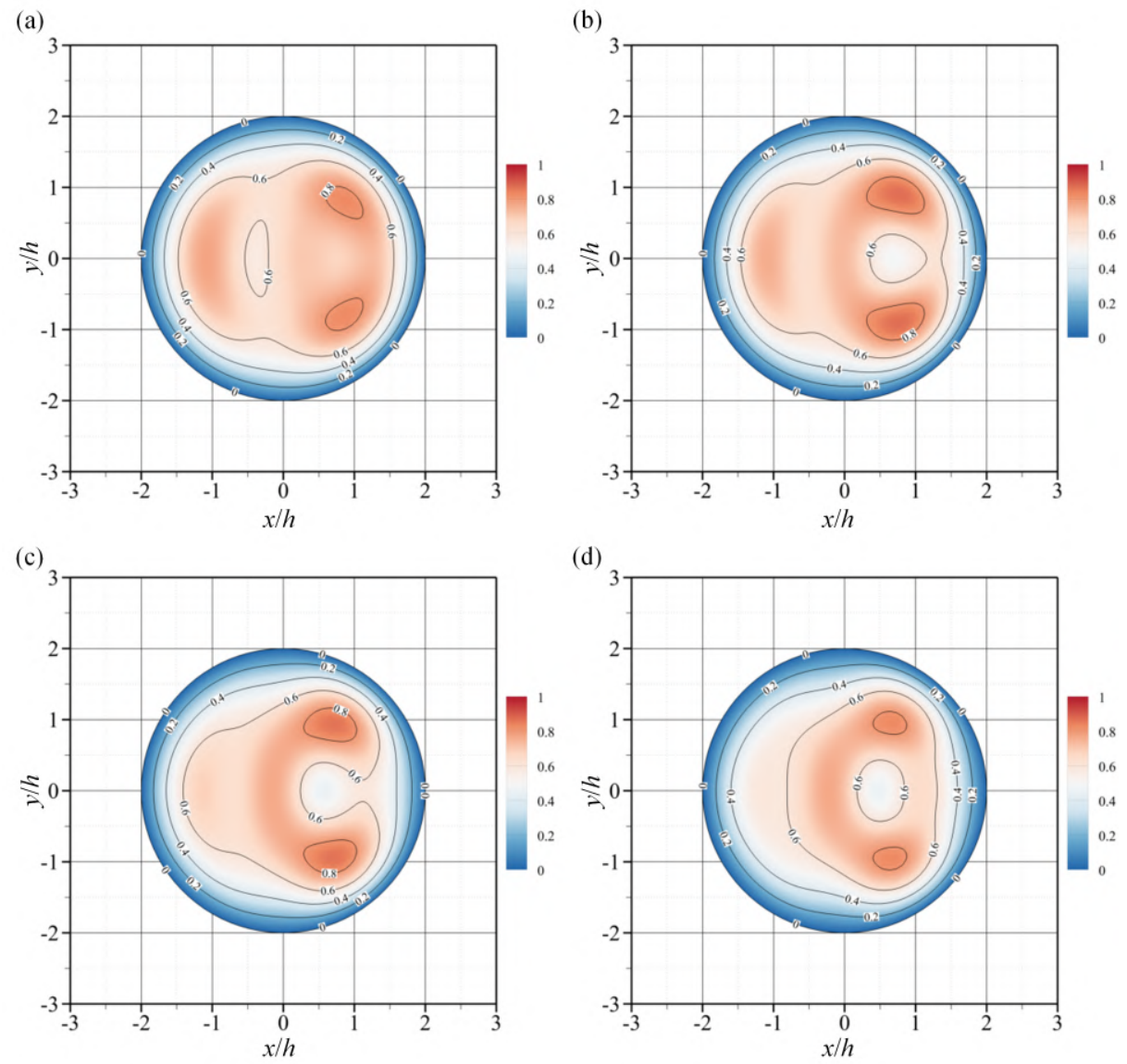

FIG. 14. Contour of the deflection of a single submerged porous elastic disk $\left|\eta_{1}\right| / A$ with different submergence: (a) $d / h=0.1$; (b) $d / h=0.15$; (c) $d / h=0.2$; (d) $d / h=0.25$. [N=1, $\bar{\alpha}=2.0, \beta=0, \bar{\chi}=\bar{\gamma}=0.01$, $R / h=2.0, \bar{P}=1.0$, simply supported]
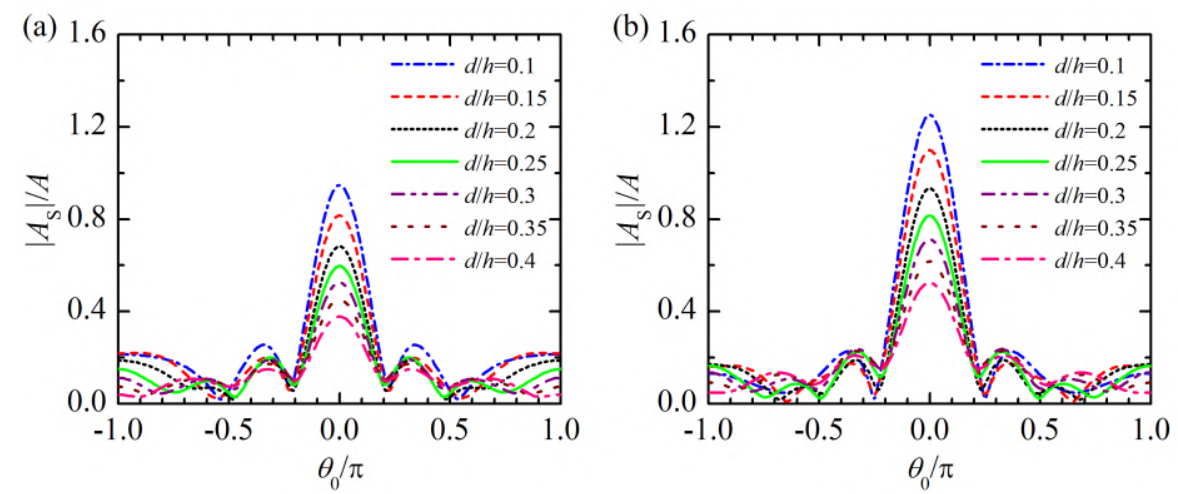

FIG. 15. Far-field scattering coefficient of a single submerged porous elastic disk with different submergence: (a) simply supported; (b) clamped. $[N=1, \bar{\alpha}=2.0, \beta=0, \bar{\chi}=\bar{\gamma}=0.01, R / h=2.0, \bar{P}=1.0]$. 
(a)

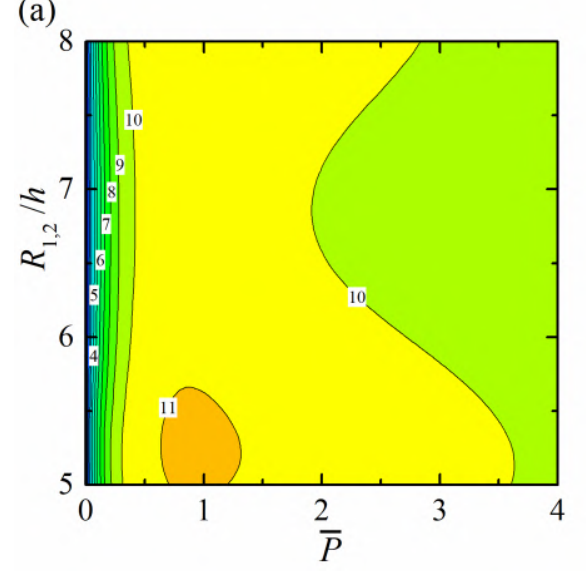

(b)

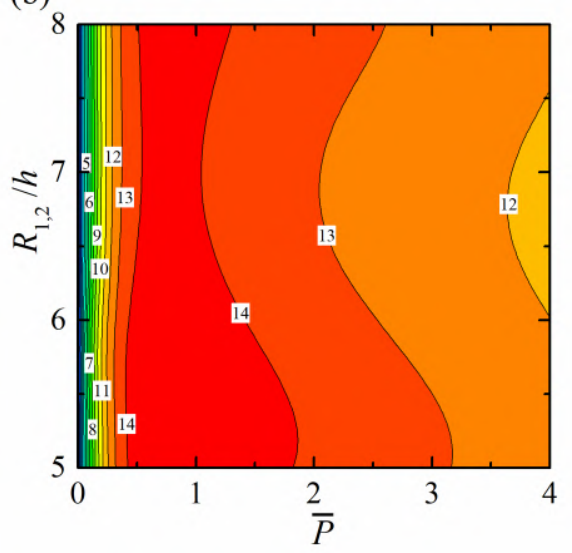

FIG. 16. Contour of wave power absorption with the distance between the disks and the porosity: (a) simply supported; (b) clamped. $\left[N=2, \bar{\alpha}=2.0, \beta=0, \bar{\chi}=\bar{\gamma}=0.01, R / h=2.0, d / h=0.2, x_{1}=x_{2}=0, y_{1}=-y_{2}\right]$
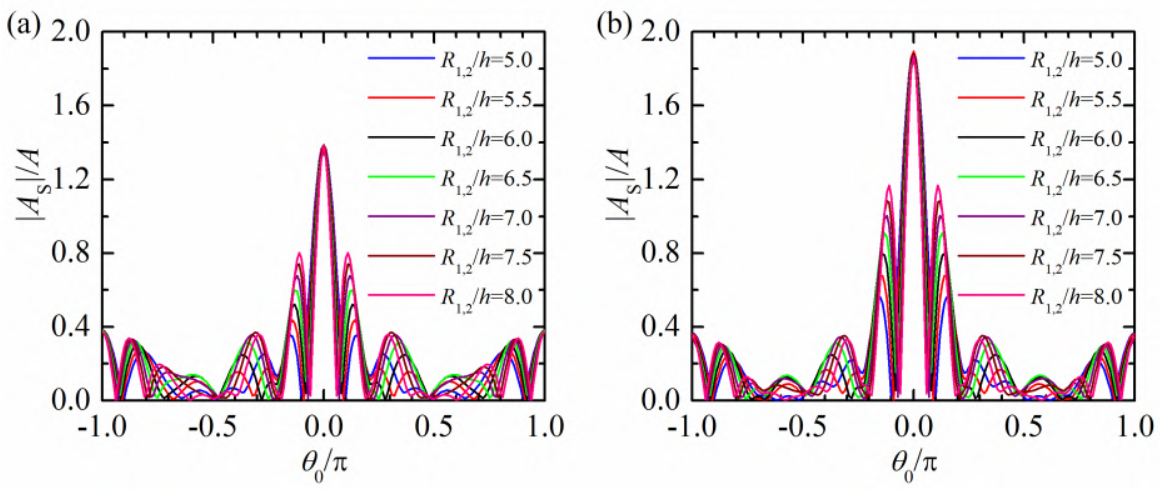

FIG. 17. Far-field scattering coefficient of a pair of submerged porous elastic disks with different distance: (a) simply supported; (b) clamped. $[N=2, \bar{\alpha}=2.0, \beta=0, \bar{\chi}=\bar{\gamma}=0.01, R / h=2.0, d / h=0.2, \bar{P}=1.0]$.

here. In the computed domain, the largest values of $|\eta| / A$ and $\left|\eta_{1}\right| / A$ are $(1.56,0.88),(1.51,0.90)$, $(1.49,0.87)$ and $(1.53,0.90)$, respectively, for these four cases tested.

Fig. 17 plots the far-field scattering coefficients of the pair of disks for different distance with $\bar{P}=1.0$. The main peak values of $\left|A_{\mathrm{S}}\right| / A$ at $\theta_{0}=0$ remain around 1.37 , and 1.87 for the simply supported and clamped edge conditions, respectively, regardless of the distance between the two submerged disks. As $R_{1,2} / h$ increases, the bandwidth of the central peak of the far-field coefficients becomes smaller. Meanwhile, the second peak, which is observed at $\theta_{0} / \pi \approx \pm 0.13$, turns higher and moves towards $\theta_{0}=0$. 
(a)

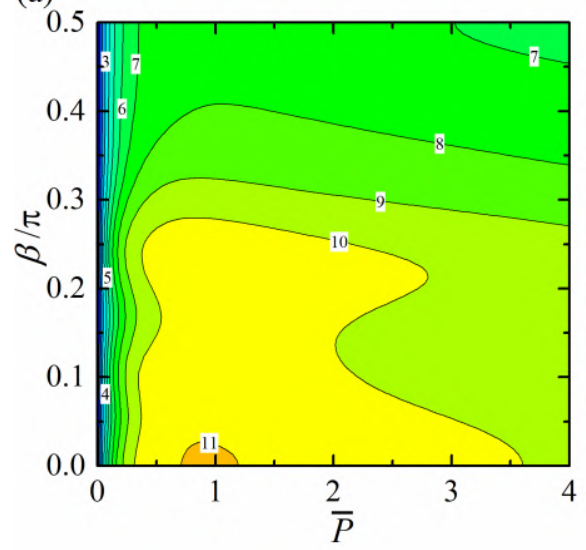

(b)

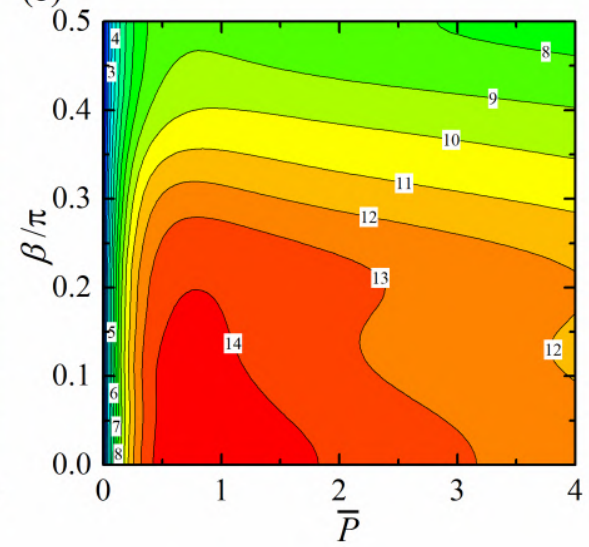

FIG. 18. Contour of wave power absorption with the incident direction and the porosity: (a) simply supported; (b) clamped. [ $N=2, \bar{\alpha}=2.0, \bar{\chi}=\bar{\gamma}=0.01, R / h=2.0, d / h=0.2, R_{1,2} / h=5.0, x_{1}=x_{2}=0$, $\left.y_{1}=-y_{2}\right]$

\section{Effect of the incident wave direction}

Fig. 18 gives the contours of the power absorption/dissipation of a pair of submerged porous elastic disks $\eta_{\text {diss }}$ versus the non-dimensional porosity parameter $\bar{P}$ and the incident wave direction $\beta$ for simply supported and clamped edge conditions. It demonstrates that the maximum wave power absorption/dissipation can be achieved when the incident waves approach from the negative $x$-axis direction ( $\beta=0$ ), i.e., with the propagating direction perpendicular to the deployment line of the two disks. As the incident waves become oblique, i.e., the incident wave direction $\beta$ increases from 0 towards $0.5 \pi$, less and less wave power can be generally absorbed/dissipated by the pair of disks, except a local peak of $\eta_{\text {diss }}$ obtained around $\beta=0.2 \pi$ with its peak value smaller than that corresponding to $\beta=0$. Taking the simply supported two disks with $\bar{P}=1.0$ as an example, $\eta_{\text {diss }}=11.04,10.37,10.44,8.81$ and 7.68 are obtained when $\beta=0, \pi / 6, \pi / 4, \pi / 3$ and $\pi / 2$, respectively, indicating a significant effect of incident wave direction on wave power absorption/dissipation of an array of submerged porous elastic disks.

The corresponding near-field wave motion and disks' deflection of these five cases are illustrated in Figs. 19 and 20, respectively. The peak of the wave amplitude $|\eta| / A$ is observed in the area right above each disk, and specifically, it is closer to the leeward side regardless of the incident wave direction. A shedding effect of the disk at the windward side on the wave motion above the other disk at the leeward side is demonstrated. This shedding effect becomes stronger 

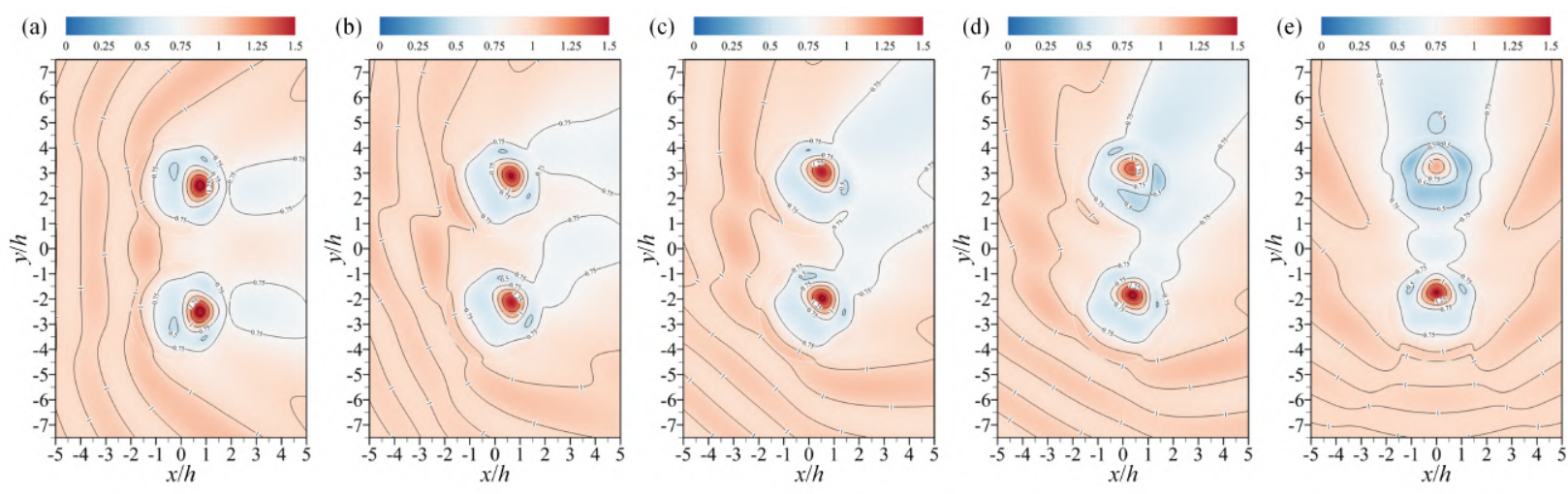

FIG. 19. Contour of the wave amplitude above a pair of submerged porous elastic disks $|\eta| / A$ with different incident wave direction: (a) $\beta=0$; (b) $\beta=\pi / 6$; (c) $\beta=\pi / 4$; (d) $\beta=\pi / 3$; (e) $\beta=\pi / 2$. [N=2, $\bar{\alpha}=2.0$, $\bar{\chi}=\bar{\gamma}=0.01, R / h=2.0, d / h=0.2, R_{1,2} / h=5.0, x_{1}=x_{2}=0, y_{1}=-y_{2}$, simply supported]

for a larger $\beta$. The peak wave amplitude above the leeward side disk gets smaller as $\beta$ increases from 0 towards $\pi / 2$. It is noted that there is a wave attenuation area of $|\eta| / A<0.75$ adjacent to the interior region and at the leeward side of each disk. In the plotted domain, with the increase of $\beta$ starting from 0 , these two areas of $|\eta| / A<0.75$ adjacent to the pair of disks' interior regions become larger and move towards each other, and ultimately merge together when $\beta=\pi / 4$. The shedding effect reaches the greatest at $\beta=\pi / 2$, for which the peak wave amplitude of $|\eta| / A$ at the interior region of the leewards side is only 1.14, and meanwhile a dramatic large wave attenuation area of $|\eta| / A<0.5$ appears at this interior region, and moreover another $|\eta| / A<0.5$ region arises at the exterior region, which is not ever observed for the remaining cases examined. The contour of the wave amplitude at the interior region of the windward side disk aligns with $\beta$, but its shape is not sensitive to the change of $\beta$. Similarly, the shedding effect on the deflection of the disks, especially the one on the leeward side, can be found in Fig. 20.

Fig. 21 illustrates the far-field scattering coefficient for different incident wave directions $\beta=$ $0, \pi / 6, \pi / 4, \pi / 3$ and $\pi / 2$ with $\bar{P}=1.0$. The direction where the main peak of $\left|A_{\mathrm{S}}\right| / A$ occurs coincides with the incident wave direction, i.e., $\theta_{0}=\beta$. The main peak value of $\left|A_{\mathrm{S}}\right| / A$ generally decreases with the increase of $\beta$ tested, except the simply supported case with $\beta=\pi / 4$, the main peak value for which, by contrast, is slightly larger than that of $\beta=\pi / 6$. Additionally, it is observed that the bandwidth of the main peak is enlarged as the incident waves propagating more likely in the direction along the line connecting the two disks' centres. 

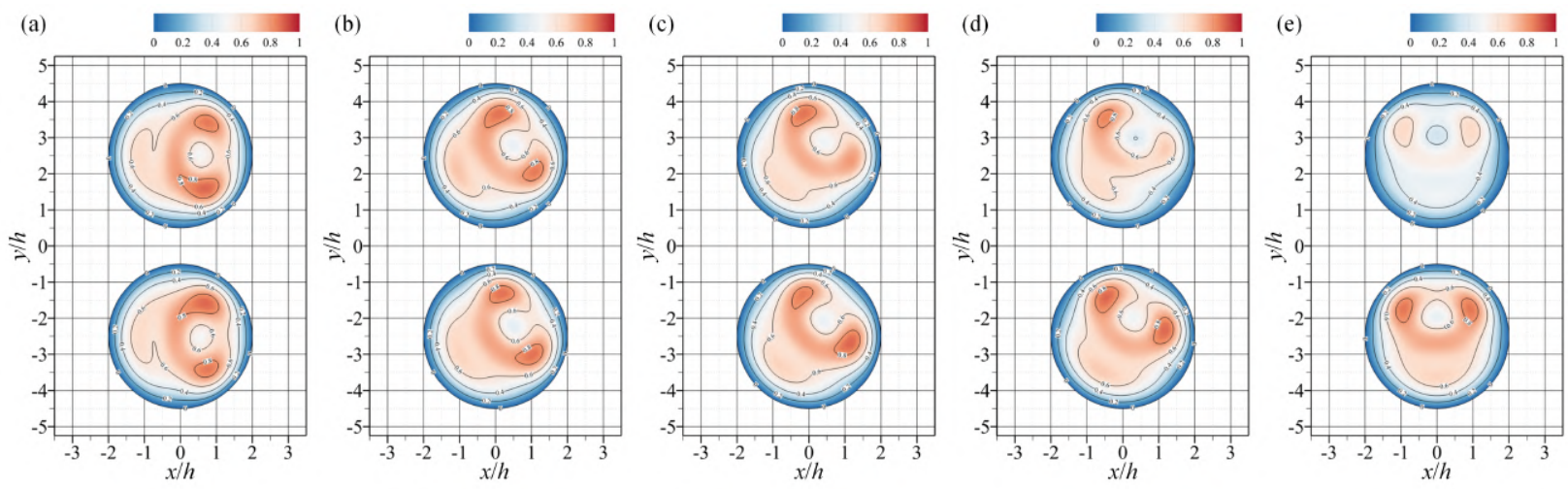

FIG. 20. Contour of the deflection of a pair of submerged porous elastic disks $\left|\eta_{n}\right| / A$ with different incident wave direction: (a) $\beta=0$; (b) $\beta=\pi / 6$; (c) $\beta=\pi / 4$; (d) $\beta=\pi / 3$; (e) $\beta=\pi / 2$. $[N=2, \bar{\alpha}=2.0$, $\bar{\chi}=\bar{\gamma}=0.01, R / h=2.0, d / h=0.2, R_{1,2} / h=5.0, x_{1}=x_{2}=0, y_{1}=-y_{2}$, simply supported]
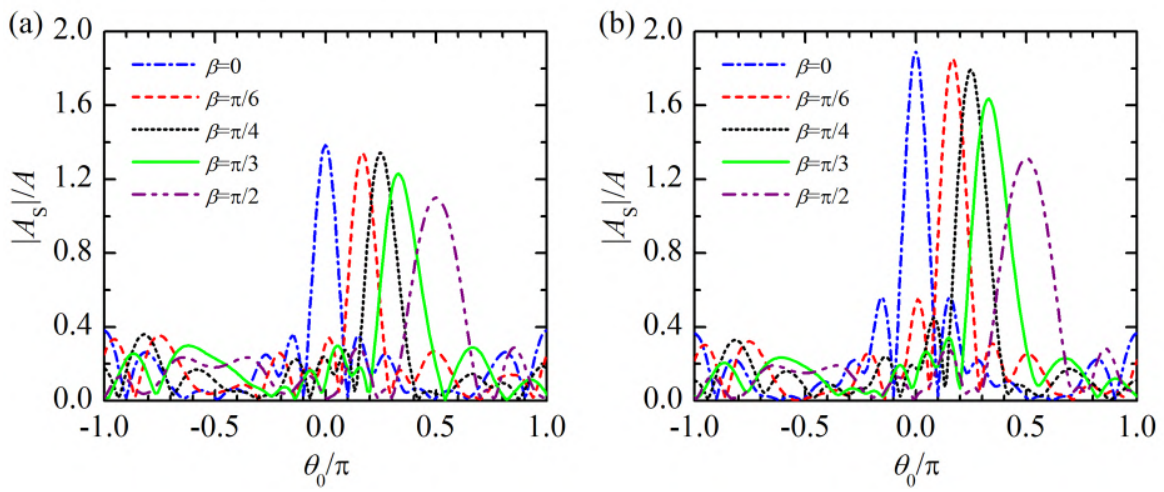

FIG. 21. Far-field scattering coefficient of a pair of submerged porous elastic disks with different incident wave directions: (a) simply supported; (b) clamped. $[N=2, \bar{\alpha}=2.0, \bar{\chi}=\bar{\gamma}=0.01, R / h=2.0, d / h=0.2$, $\left.R_{1,2} / h=5.0, \bar{P}=1.0\right]$.

\section{Effect of the number of disks}

In order to provide a significant contribution to wave power absorption/dissipation, an array of multiple submerged, porous elastic disks should be deployed.

Fig. 22 presents the variation of wave power absorption/dissipation in terms of $\eta_{\text {diss }} / N$ for an array consisting of $N(N=1,2, \cdots, 5)$ disks against $\bar{P}$ with $\bar{\alpha}=2.0, \beta=0$. In the computed range of $\bar{P}$, the more disks included in the array, the larger the value of $\eta_{\text {diss }} / N$, demonstrating a constructive hydrodynamic interaction for wave power absorption/dissipation. The most significant improvement of $\eta_{\text {diss }} / N$ occurs when $N$ increases from 1 to 2 . For larger values of $N$, the increase 
(a)

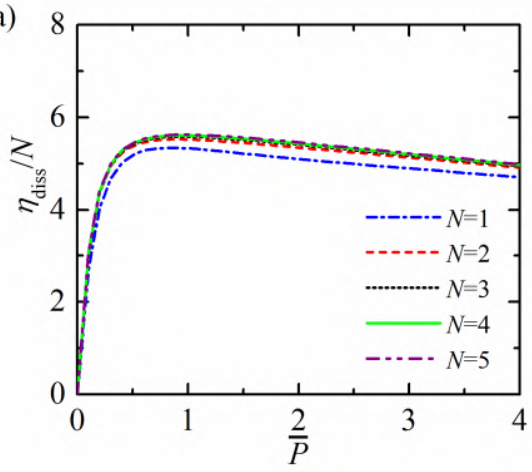

(b)

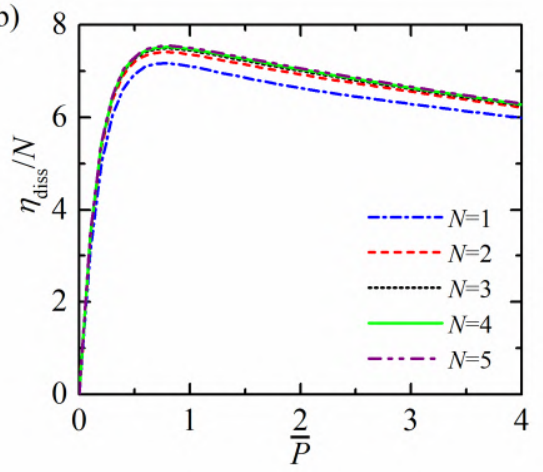

FIG. 22. Variation of wave power absorption/dissipation of an array of submerged porous elastic disks consisting of different number of disks with different porosity: (a) simply supported; (b) clamped. $[\bar{\alpha}=2.0$, $\left.\beta=0, R / h=2.0, d / h=0.2, R_{j, j+1} / h=5.0, \bar{\chi}=\bar{\gamma}=0.01\right]$.
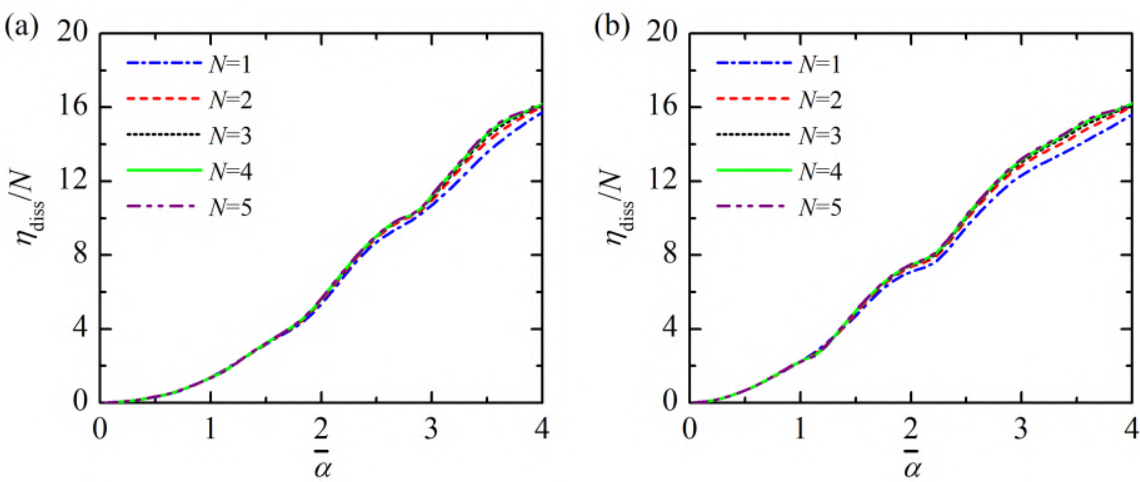

FIG. 23. Frequency response of wave power absorption/dissipation of an array of submerged porous elastic disks consisting of different number of disks: (a) simply supported; (b) clamped. $[\bar{\alpha}=2.0, \beta=0, R / h=$ $\left.2.0, d / h=0.2, R_{j, j+1} / h=5.0, \bar{\chi}=\bar{\gamma}=0.01, \bar{P}=1.0\right]$.

in $\eta_{\text {diss }} / N$ is weaker.

The frequency response of $\eta_{\text {diss }} / N$ for the array with $\bar{P}=1.0$ is given in Fig. 23. For long waves, e.g., $\bar{\alpha}<1.5$, the curves almost overlap one another, representing a disk-number independent $\eta_{\text {diss }} / N$ for these wave conditions. For the other wave conditions, especially $\bar{\alpha} \in(2.5,4.0]$ and $(2.0,4.0]$ for the simply supported and clamped edge conditions, respectively, a larger value of $\eta_{\text {diss }} / N$ can be obtained by the array with more disks included. Compared to enlarging the area of a single submerged porous elastic disk (see Figs. 7 and 8), deploying multiple disks in an array (Figs. 22 and 23) is demonstrated to be a more promising method for wave power absorption/dissipation. 

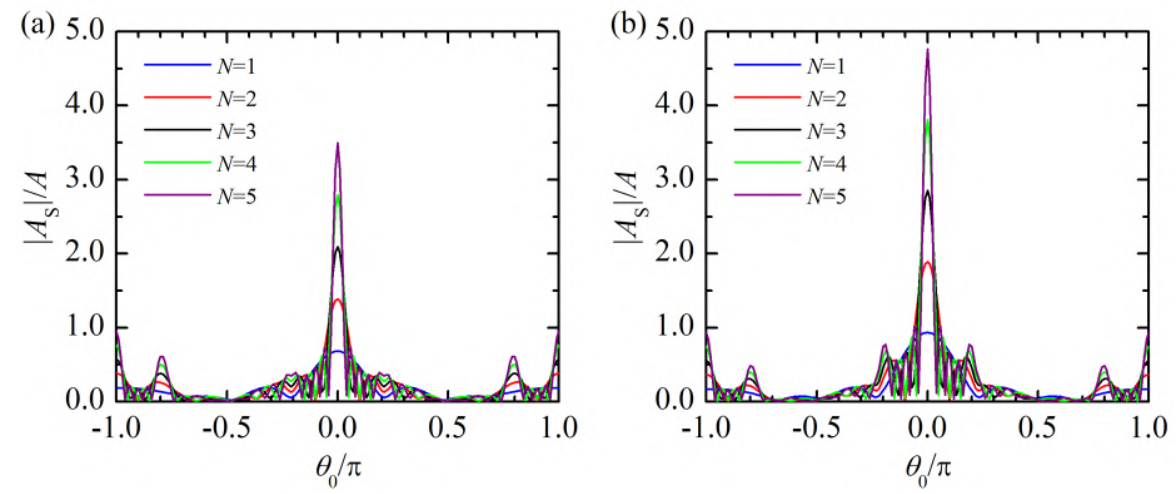

FIG. 24. Far-field scattering coefficient of an array of submerged porous elastic disks consisting of different number of disks: (a) simply supported; (b) clamped. $[N=2, \bar{\alpha}=2.0, \bar{\chi}=\bar{\gamma}=0.01, R / h=2.0, d / h=0.2$, $\left.R_{j, j+1} / h=5.0, \bar{P}=1.0\right]$.

The near-field wave motion and the disks' deflection for a different number of disks in the array are examined as well. The contours of $|\eta| / A$ at the interior regions and the $\left|\eta_{n}\right| / A$ are not sensitive upon the change of $N$. Hence the results are not given here to save the space.

The effect of the number of disks $N$ on the far-field scattering coefficient is also studied, the results of which are plotted in Fig. 24. The more disks included in the array, the more fluctuations of the $\left|A_{S}\right| / A-\theta_{0}$ curve observed. The values of $\left|A_{S}\right| / A$ at $\theta=0, \pm 0.2 \pi, \pm 0.8 \pi$ and $\pm 1.0 \pi$ are found to be dependent on $N$ dramatically. Their values change proportionally with the change of $N$, whereas the value of $\left|A_{\mathrm{S}}\right| / A$ vanishes around $\theta= \pm 0.5 \pi$ regardless of the number of disks. A similar pattern was reported by Kar, Koley, and Sahoo (2018) in the case of wave scattering of surface gravity waves over multiple trenches and explained in terms of Bragg scattering, which is one of the interesting physical phenomena related to surface wave propagation over periodic sand bars/ripples or multiple floating/submerged floats (Kar, Sahoo, and Behera, 2019).

\section{CONCLUSIONS}

A theoretical model based on linear potential flow theory and the eigenfunction matching method is developed in this paper to study the hydroelastic response of submerged porous elastic discs, which may represent a wave farm consisting of elastic plate-shaped WECs to absorb wave power, or a flexible submerged elastic breakwater to attenuate ocean waves. Apart from evaluating the power absorbed/dissipated by the submerged porous elastic discs with a straightfor- 
ward method, an indirect method with the employment of the Kochin functions is derived based on Green's theorem. We find that the indirect method provides a similar level of accuracy as the direct method, at a fraction of the computational cost.

The proposed theoretical model was validated by comparing the present results with the published data. The direct- and indirect methods were both applied to calculate wave power absorption/dissipation of a pair of submerged elastic discs, which were found in an excellent agreement. Upon validation, the theoretical model was applied to investigate the performance of a single submerged porous elastic disc first, and then an array of discs. A multi-parameter impact analysis on wave power absorption/dissipation, near-field wave motion, disc deflection and the far-field scattering coefficient was carried out.

The following conclusions may be drawn:

(i) For a single submerged porous elastic disc or an array of discs subjected to regular waves, there is a proper value of porosity parameter to maximise the wave power absorption/dissipation. The largest surface wave motion occurs at the region right above the submerged discs. The near-field wave motion and the disc deflection can be dramatically weakened by increasing the porosity of the disc.

(ii) Enlarging the radius of a submerged porous elastic disc generally results in less wave power that can be absorbed/dissipated per unit area of the disc. The patterns of the near-field wave motion and the disc deflection vary significantly with the change of disc radius.

(iii) For long waves, the disc with its edge clamped shows a better performance in wave power absorption/dissipation compared to that with a simply supported edge. Whereas for short waves, the advantage of the clamped edge is not obvious, and for some particular circumstances, a simply supported edge condition is even more conducive to wave power absorption/dissipation.

(iv) For a single porous elastic disc with large porosity parameter, the wave power absorbed/dissipated by the disc decreases with the increase of the disc submergence. However, for a porous elastic disc with certain values of porosity parameter, the largest wave power absorption/dissipation is obtained when the disc is submerged at a critical submergence which is not too shallow. 
(v) For a pair of submerged porous elastic discs subjected to waves propagating perpendicular to the deployment line of the discs, there is a proper distance between the discs that can maximise the wave power absorption/dissipation. The main peak of the far-field scattering coefficient remains at a certain value regardless of the distance between the two discs. Nevertheless, the main peak bandwidth becomes smaller with the increase of the discs' distance.

(vi) When the incoming wave direction is aligned with the deployment line of the discs, the least wave power can be absorbed/dissipated because of the shedding effect of the disc at the windward side on the other one at the leeward side. The most wave power can be absorbed/dissipated when the wave incident direction is perpendicular to the deployment line of the discs.

(vii) The more discs included in an array, the larger the power that can be absorbed/dissipated per disc on average due to the constructive hydrodynamic interaction between them. The far-field scattering coefficients at some specified directions are found to be dramatically dependent on the number of discs in the array; whereas, for some of the other directions, the coefficients vanish regardless of the number of discs.

The present theoretical model is developed in the framework of potential flow theory; hence it may not be suitable for extreme wave-structure interactions. 'Bragg scattering' and 'wavetrapping'/'near-trapping' effects as described by, e.g., Kar, Koley, and Sahoo (2018), Sahoo, Lee, and Chwang (2000) and Thompson, Linton, and Porter (2008) were not registered in our results. However, these are aspects of interest that we plan to investigate as a continuation of this line of research.

Data availability statement. The key data to fully reproduce the results of this work are presented in table 1.

\section{ACKNOWLEDGMENTS}

The research was supported by Intelligent Community Energy (ICE), INTERREG V FCE, European Commission (Contract No. 5025) and Open Research Fund Program of State Key Laboratory of Hydraulic Engineering Simulation and Safety, Tianjin University (Grant No. HESS-1902). 


\section{Appendix A: Derivation process of the formulas and calculation for the unknown} coefficients $A_{m, l}^{(n)}$ and $B_{m, l}^{(n)}$

Here we take the case with simply supported edge condition as an example to show how to determine the unknown coefficients $A_{m, l}^{(n)}$ and $B_{m, l}^{(n)}$. Inserting the expression of the spatial potentials for both the exterior and interior regions, i.e., Eqs. (16)-(17), into continuity conditions at the interfaces and the simply supported edge boundary conditions, Eqs. (11) and (21)-(22), gives:

$$
\begin{aligned}
& -\frac{\mathrm{i} g A}{\omega} Z_{0}(z) \mathrm{e}^{\mathrm{i} k\left(x_{n} \cos \beta+y_{n} \sin \beta\right)} \sum_{m=-\infty}^{\infty} \mathrm{i}^{m} \mathrm{e}^{-\mathrm{i} m \beta} J_{m}\left(k R_{n}\right) \mathrm{e}^{\mathrm{i} m \theta_{n}}+\sum_{m=-\infty}^{\infty} \sum_{l=0}^{\infty} A_{m, l}^{(n)} H_{m}\left(k_{l} R_{n}\right) Z_{l}(z) \mathrm{e}^{\mathrm{i} m \theta_{n}} \\
& +\sum_{\substack{j=1, m \\
j \neq n}}^{N} \sum_{m=-\infty}^{\infty} \sum_{l=0}^{\infty} A_{m, l}^{(j)} Z_{l}(z) \sum_{m^{\prime}=-\infty}^{\infty}(-1)^{m^{\prime}} H_{m-m^{\prime}}\left(k_{l} R_{n, j}\right) J_{m^{\prime}}\left(k_{l} R_{n}\right) \mathrm{e}^{\mathrm{i}\left(m \alpha_{j, n}-m^{\prime} \alpha_{n, j}\right)} \mathrm{e}^{\mathrm{i} m^{\prime} \theta_{n}} \\
& =\sum_{m=-\infty}^{\infty} \sum_{l=-2}^{\infty} B_{m, l}^{(n)} I_{m}\left(\kappa_{l}^{(n)} R_{n}\right) Y_{l}^{(n)}(z) \mathrm{e}^{\mathrm{i} m \theta_{n}}, \quad-h<z<0, \\
& -\frac{\mathrm{i} g A}{\omega} Z_{0}(z) \mathrm{e}^{\mathrm{i} k\left(x_{n} \cos \beta+y_{n} \sin \beta\right)} \sum_{m=-\infty}^{\infty} \mathrm{i}^{m} \mathrm{e}^{-\mathrm{i} m \beta} k J_{m}^{\prime}\left(k R_{n}\right) \mathrm{e}^{\mathrm{i} m \theta_{n}}+\sum_{m=-\infty}^{\infty} \sum_{l=0}^{\infty} A_{m, l}^{(n)} k_{l} H_{m}^{\prime}\left(k_{l} R_{n}\right) Z_{l}(z) \mathrm{e}^{\mathrm{i} m \theta_{n}} \\
& +\sum_{\substack{j=1, m \\
j \neq n}}^{N} \sum_{m=-\infty}^{\infty} \sum_{l=0}^{\infty} A_{m, l}^{(j)} k_{l} Z_{l}(z) \sum_{m^{\prime}=-\infty}^{\infty}(-1)^{m^{\prime}} H_{m-m^{\prime}}\left(k_{l} R_{n, j}\right) J_{m^{\prime}}^{\prime}\left(k_{l} R_{n}\right) \mathrm{e}^{\mathrm{i}\left(m \alpha_{j, n}-m^{\prime} \alpha_{n, j}\right)} \mathrm{e}^{\mathrm{i} m^{\prime} \theta_{n}} \\
& =\sum_{m=-\infty}^{\infty} \sum_{l=-2}^{\infty} B_{m, l}^{(n)} \kappa_{l}^{(n)} I_{m}^{\prime}\left(\kappa_{l}^{(n)} R_{n}\right) Y_{l}^{(n)}(z) \mathrm{e}^{\mathrm{i} m \theta_{n}}, \quad-h<z<0 \\
& \sum_{m=-\infty}^{\infty} \sum_{l=-2}^{\infty} \frac{\kappa_{l}^{(n)} \sin \left(\kappa_{l}^{(n)} h\right)+\frac{\omega^{2}}{g} \cos \left(\kappa_{l}^{(n)} h\right)}{\chi_{n}\left(\kappa_{l}^{(n)}\right)^{4}-\frac{\omega^{2}}{g} \gamma_{n}} B_{m, l}^{(n)} f_{M}(n, m, l) \mathrm{e}^{\mathrm{i} m \theta_{n}}=0 \\
& \sum_{m=-\infty}^{\infty} \sum_{l=-2}^{\infty} \frac{\kappa_{l}^{(n)} \sin \left(\kappa_{l}^{(n)} h\right)+\frac{\omega^{2}}{g} \cos \left(\kappa_{l}^{(n)} h\right)}{\chi_{n}\left(\kappa_{l}^{(n)}\right)^{4}-\frac{\omega^{2}}{g} \gamma_{n}} B_{m, l}^{(n)} I_{m}\left(\kappa_{l}^{(n)} R_{n}\right) \mathrm{e}^{\mathrm{i} m \theta_{n}}=0
\end{aligned}
$$

where

$$
f_{M}(n, m, l)=R_{n}^{2}\left(\kappa_{l}^{(n)}\right)^{2} I_{m}^{\prime \prime}\left(\kappa_{l}^{(n)} R_{n}\right)-m^{2} v I_{m}\left(\kappa_{l}^{(n)} R_{n}\right)+R_{n} \kappa_{l} v I_{m}^{\prime}\left(\kappa_{l}^{(n)} R_{n}\right)
$$

After multiplying both sides of Eqs. (A1)-(A2) with $Z_{\zeta}(z) \mathrm{e}^{-\mathrm{i} \tau \theta_{n}}$ and integrating in $z \in[-h, 0]$ and $\theta_{n} \in[0,2 \pi]$ and using the orthogonality characteristics of them, Eqs. (A1)-(A2) can be rewritten as 


$$
\begin{gathered}
A_{\tau, \zeta}^{(n)} H_{\tau}\left(k_{\zeta} R_{n}\right) A_{\zeta}+\sum_{\substack{j=1, m=-\infty \\
j \neq n}}^{N} \sum_{m, \zeta}^{\infty} A_{\zeta}^{(j)}(-1)^{\tau} H_{m-\tau}\left(k_{\zeta} R_{n, j}\right) J_{\tau}\left(k_{\zeta} R_{n}\right) \mathrm{e}^{\mathrm{i}\left(m \alpha_{j, n}-\tau \alpha_{n, j}\right)} \\
-\sum_{l=-2}^{\infty} B_{\tau, l}^{(n)} I_{\tau}\left(\kappa_{l}^{(n)} R_{n}\right) Y_{l, \zeta}^{(n)}=\frac{\mathrm{i} g A}{\omega} \delta_{0, \zeta} A_{\zeta} \mathrm{e}^{\mathrm{i} k\left(x_{n} \cos \beta+y_{n} \sin \beta\right)} \mathrm{i}^{\tau} \mathrm{e}^{-\mathrm{i} \tau \beta} J_{\tau}\left(k R_{n}\right), \\
A_{\tau, \zeta}^{(n)} k_{\zeta} H_{\tau}^{\prime}\left(k_{\zeta} R_{n}\right) A_{\zeta}+\sum_{\substack{j=1, m=-\infty \\
j \neq n}}^{N} \sum_{m, \zeta}^{\infty} A_{\zeta}(-1)^{\tau} H_{m-\tau}\left(k_{\zeta} R_{n, j}\right) k_{\zeta} J_{\tau}^{\prime}\left(k_{\zeta} R_{n}\right) \mathrm{e}^{\mathrm{i}\left(m \alpha_{j, n}-\tau \alpha_{n, j}\right)} \\
-\sum_{l=-2}^{\infty} B_{\tau, l}^{(n)} \kappa_{l}^{(n)} I_{\tau}^{\prime}\left(\kappa_{l}^{(n)} R_{n}\right) Y_{l, \zeta}^{(n)}=\frac{\mathrm{i} g A}{\omega} \delta_{0, \zeta} A \zeta \mathrm{e}^{\mathrm{i} k\left(x_{n} \cos \beta+y_{n} \sin \beta\right)_{\mathrm{i}} \tau} \mathrm{e}^{-\mathrm{i} \tau \beta} k J_{\tau}^{\prime}\left(k R_{n}\right),
\end{gathered}
$$

where

$$
\begin{gathered}
A_{l}=\int_{-h}^{0} Z_{l}^{2}(z) \mathrm{d} z=\frac{\sinh \left(k_{l} h\right) \cosh \left(k_{l} h\right)+k_{l} h}{2 k_{l} \cosh ^{2}\left(k_{l} h\right)}, \\
Y_{l, \zeta}^{(n)}=\int_{-h}^{0} Y_{l}^{(n)}(z) Z_{\zeta}(z) \mathrm{d} z=\frac{k_{\zeta} h \sinh \left(k_{\zeta} c_{n}\right)\left[\frac{\omega^{2}}{g} \cos \left(\kappa_{l}^{(n)} h\right)+\kappa_{l}^{(n)} \sin \left(\kappa_{l}^{(n)} h\right)\right]}{\cosh \left(k_{\zeta} h\right)\left(\left(\kappa_{l}^{(n)}\right)^{2}+k_{\zeta}^{2}\right)} .
\end{gathered}
$$

In a similar way, after multiplying both sides of Eqs. (A3)-(A4) with $\mathrm{e}^{-\mathrm{i} \tau \theta_{n}}$ and integrating in $\theta_{n} \in[0,2 \pi]$, Eqs. (A3)-(A4) can be rewritten as

$$
\begin{aligned}
& \sum_{l=-2}^{\infty} \frac{\kappa_{l}^{(n)} \sin \left(\kappa_{l}^{(n)} h\right)+\frac{\omega^{2}}{g} \cos \left(\kappa_{l}^{(n)} h\right)}{\chi_{n}\left(\kappa_{l}^{(n)}\right)^{4}-\frac{\omega^{2}}{g} \gamma_{n}} B_{\tau, l}^{(n)} f_{M}(n, \tau, l)=0, \\
& \sum_{l=-2}^{\infty} \frac{\kappa_{l}^{(n)} \sin \left(\kappa_{l}^{(n)} h\right)+\frac{\omega^{2}}{g} \cos \left(\kappa_{l}^{(n)} h\right)}{\chi_{n}\left(\kappa_{l}^{(n)}\right)^{4}-\frac{\omega^{2}}{g} \gamma_{n}} B_{\tau, l}^{(n)} I_{\tau}\left(\kappa_{l}^{(n)} R_{n}\right)=0 .
\end{aligned}
$$

In order to evaluate the unknown coefficients $A_{m, l}^{(n)}$ and $B_{m, l}^{(n)}$, we truncate all infinite series of vertical eigenfunctions at $L$, i.e., $(L+1)$ terms $(l=0,1, \ldots, L)$ for $A_{m, l}^{(n)}$ and $(L+3)$ terms $(l=$ $-2,-1,0,1, \ldots, L)$ for $B_{m, l}^{(n)}$, and we take $(2 M+1)$ angular terms $(m=-M, \ldots, 0, \ldots, M)$, resulting in $2 N(2 M+1)(L+2)$ unknown coefficients to be determined. After taking $(\tau=-M, \ldots, 0, \ldots, M)$ and $(\zeta=0,1, \ldots, L)$ in Eqs. (A6)-(A7) and (A10)-(A11), a $2 N(2 M+1)(L+2)$ order complex linear equation matrix is obtained, which can be used to determine the exact same number of unknown coefficients. $M$ and $L$ should be chosen large enough to lead to accurate results. In all the theoretical computations as given in this paper, $M=10$ and $L=20$ are used, unless otherwise specified. 


\section{Appendix B: Derivation process of the energy identities}

In the fluid domain enclosed by $\Omega_{R}$, free water surface and the sea bed, but with the volume occupied by the $N$ discs excluded, using Green's theorem (Falnes, 2002), we have

$$
\begin{aligned}
& \oiint\left(\phi \frac{\partial \phi^{*}}{\partial n}-\phi^{*} \frac{\partial \phi}{\partial n}\right) \mathrm{d} s \\
& =\sum_{n=1}^{N} \iint_{\Omega_{n}}\left[\left(\phi_{-} \frac{\partial \phi_{-}^{*}}{\partial z}-\phi_{-}^{*} \frac{\partial \phi_{-}}{\partial z}\right)-\left(\phi_{+} \frac{\partial \phi_{+}^{*}}{\partial z}-\phi_{+}^{*} \frac{\partial \phi_{+}}{\partial z}\right)\right] \mathrm{d} s+\iint_{\Omega_{R}}\left(\phi \frac{\partial \phi^{*}}{\partial r}-\phi^{*} \frac{\partial \phi}{\partial r}\right) \mathrm{d} s=0 .
\end{aligned}
$$

With utilization of Eq. (5), Eq. (B1) can be rewritten as

$$
\sum_{n=1}^{N} \iint_{\Omega_{n}}\left[\mathrm{i} \omega\left(\left(\phi_{-}-\phi_{+}\right) \eta_{n}^{*}+\left(\phi_{-}^{*}-\phi_{+}^{*}\right) \eta_{n}\right)-2 \mathrm{i} P_{n}\left|\phi_{-}-\phi_{+}\right|^{2}\right] \mathrm{d} s=-\iint_{\Omega_{R}}\left(\phi \frac{\partial \phi^{*}}{\partial r}-\phi^{*} \frac{\partial \phi}{\partial r}\right) \mathrm{d} s .
$$

The deflection of the $n$-th disc might be expressed by a series of modes as (Meylan, Bennetts, and Peter, 2017)

$$
\eta_{n}=\sum_{q=1}^{Q} u_{q}^{(n)} \eta_{q}^{(n)}
$$

where the modes $\eta_{q}^{(n)}$ satisfy the eigenvalue problem for the biharmonic operator

$$
\Delta^{2} \eta_{q}^{(n)}=\lambda_{q} \eta_{q}^{(n)}
$$

together with the edge conditions as given in $\S-\mathrm{II} ; \eta_{q}^{(n)}$ are orthogonal for different eigenvalues $\lambda_{q}$, and $Q$ denotes the truncated numbers of the infinite modes.

The dynamic motion of the discs can be coupled with the hydrodynamics by

$$
\left(\mathbf{K}+\mathbf{C}-\frac{\omega^{2}}{g} \mathbf{M}\right) \boldsymbol{u}=\mathrm{i} \omega \rho \iint_{\Omega_{\text {sum }}}\left(\phi_{-}-\phi_{+}\right) \boldsymbol{n} \mathrm{d} s,
$$

where $\Omega_{\text {sum }}=\Omega_{1} \cup \Omega_{2} \cup \ldots \cup \Omega_{N}, \mathbf{K}, \mathbf{C}$ and $\mathbf{M}$ are $(N Q) \times(N Q)$ square matrices that represent stiffness, hydrostatic-restoring and mass matrices, respectively,

$$
\mathbf{K}=\left\langle\chi \lambda_{q}\right\rangle_{(n-1) Q+q} ; \quad \mathbf{C}=\mathbf{I} ; \quad \mathbf{M}=\gamma \mathbf{I},
$$

in which $\left\langle c_{i}\right\rangle_{j}$ denotes a diagonal matrix with diagonal entries $c_{i}$ at the position $(j, j), \mathbf{I}$ is the identity matrix, and

$$
\boldsymbol{u}=\left[u_{q}^{(n)}\right]_{(n-1) Q+q}, \quad \boldsymbol{n}=\left[\eta_{q}^{(n)}\right]_{(n-1) Q+q}
$$


where $\left[c_{i}\right]_{j}$ represents a vector with entries $c_{i}$ at the $j$-th row.

With the employment of Eqs. (B3) and (B5), it can be proved that

$$
\begin{aligned}
& \sum_{n=1}^{N} \iint_{\Omega_{n}}\left(\left(\phi_{-}-\phi_{+}\right) \eta_{n}^{*}+\left(\phi_{-}^{*}-\phi_{+}^{*}\right) \eta_{n}\right) \mathrm{d} s=\sum_{n=1}^{N} \iint_{\Omega_{n}}\left(\left(\phi_{-}-\phi_{+}\right) \sum_{q=1}^{Q} u_{q}^{(n) *} \eta_{q}^{(n)}+\left(\phi_{-}^{*}-\phi_{+}^{*}\right) \sum_{q=1}^{Q} u_{q}^{(n)} \eta_{q}^{(n)}\right) \\
= & \iint_{\Omega_{\mathrm{sum}}}\left(-\left(\phi_{-}(\mathbf{x})-\phi_{+}(\mathbf{x})\right)\left[\left(\mathbf{K}+\mathbf{C}-\frac{\omega^{2}}{g} \mathbf{M}\right)^{-1} \mathrm{i} \omega \rho \iint_{\Omega_{\mathrm{sum}}}\left(\phi_{-}^{*}(\overline{\mathbf{x}})-\phi_{+}^{*}(\overline{\mathbf{x}})\right) \boldsymbol{n}(\overline{\mathbf{x}}) \mathrm{d} \bar{s}\right]^{\mathrm{T}} \boldsymbol{n}(\mathbf{x})\right. \\
& \left.+\left(\phi_{-}^{*}(\mathbf{x})-\phi_{+}^{*}(\mathbf{x})\right)\left[\left(\mathbf{K}+\mathbf{C}-\frac{\omega^{2}}{g} \mathbf{M}\right)^{-1} \mathrm{i} \omega \rho \iint_{\Omega_{\mathrm{sum}}}\left(\phi_{-}(\overline{\mathbf{x}})-\phi_{+}(\overline{\mathbf{x}})\right) \boldsymbol{n}(\overline{\mathbf{x}}) \mathrm{d} \bar{s}\right]^{\mathrm{T}} \boldsymbol{n}(\mathbf{x})\right) \mathrm{d} s=0
\end{aligned}
$$

where we used the symmetry of the matrix $\left(\mathbf{K}+\mathbf{C}-\frac{\omega^{2}}{g} \mathbf{M}\right)^{-1}$ and reversed the order of integration (Flavià and Meylan, 2019).

Therefore, Eq. (B2) reads

$$
\sum_{n=1}^{N} \iint_{\Omega_{n}}\left(-2 \mathrm{i} P_{n}\left|\phi_{-}-\phi_{+}\right|^{2}\right) \mathrm{d} s=-\iint_{\Omega_{R}}\left(\phi \frac{\partial \phi^{*}}{\partial r}-\phi^{*} \frac{\partial \phi}{\partial r}\right) \mathrm{d} s
$$

hence the power absorption/dissipation can be expressed as

$$
P_{\mathrm{diss}}=\frac{\rho \omega}{2} \sum_{n=1}^{N} P_{n} \iint_{\Omega_{n}}\left|\phi_{-}-\phi_{+}\right|^{2} \mathrm{~d} s=\frac{\rho \omega}{4 \mathrm{i}} \iint_{\Omega_{R}}\left(\phi \frac{\partial \phi^{*}}{\partial r}-\phi^{*} \frac{\partial \phi}{\partial r}\right) \mathrm{d} s .
$$

\section{REFERENCES}

Abramowitz, M. and Stegun, I. A., Handbook of mathematical functions (Government Printing Office, Washington, D.C., 1964).

Ahmadian, A. S., "Chapter 1 - introduction," in Numerical models for submerged breakwaters, edited by A. S. Ahmadian (Butterworth-Heinemann, Boston, 2016) pp. 1-15.

Behera, H. and Sahoo, T., "Hydroelastic analysis of gravity wave interaction with submerged horizontal flexible porous plate," Journal of Fluids and Structures 54, 643-660 (2015).

Buriani, F. and Renzi, E., "Hydrodynamics of a flexible piezoelectric wave energy harvester moored on a breakwater," in 12th European Wave and Tidal Energy Conference (EWTEC) (Cork, Ireland, 2017).

Cho, I. H. and Kim, M. H., "Interactions of horizontal porous flexible membrane with waves," Journal of Waterway, Port, Coastal, and Ocean Engineering 126, 245-253 (2000). 
Cho, I. H., Koh, H. J., Kim, J. R., and Kim, M. H., "Wave scattering by dual submerged horizontal porous plates," Ocean Engineering 73, 149-158 (2013).

Chwang, A. T. and Wu, J., "Wave scattering by submerged porous disk," Journal of Engineering Mechanics 120, 2575-2587 (1994).

Evans, D. and Porter, R., "Hydrodynamic characteristics of an oscillating water column device," Applied Ocean Research 17, 155 - 164 (1995).

Evans, D. V. and Peter, M. A., "Asymptotic reflection of linear water waves by submerged horizontal porous plates,” Journal of Engineering Mathematics 69, 135-154 (2011).

Falnes, J., Ocean waves and oscillating systems: linear interactions including wave-energy extraction (Cambridge university press, 2002).

Fang, Z., Xiao, L., and Peng, T., "Generalized analytical solution to wave interaction with submerged multi-layer horizontal porous plate breakwaters," Journal of Engineering Mathematics 105, 117-135 (2017).

Farina, L. and Martin, P., "Scattering of water waves by a submerged disc using a hypersingular integral equation,” Applied Ocean Research 20, 121-134 (1998).

Flavià, F. F. and Meylan, M. H., “An extension of general identities for 3d water-wave diffraction with application to the diffraction transfer matrix," Applied Ocean Research 84, 279-290 (2019).

Islam, N., Kundu, S., and Gayen, R., "Scattering and radiation of water waves by a submerged rigid disc in a two-layer fluid," Proceedings of the Royal Society A: Mathematical, Physical and Engineering Sciences 475, 20190331 (2019).

Kar, P., Koley, S., and Sahoo, T., "Scattering of surface gravity waves over a pair of trenches," Applied Mathematical Modelling 62, 303-320 (2018).

Kar, P., Sahoo, T., and Behera, H., "Effect of bragg scattering due to bottom undulation on a floating dock," Wave Motion 90, 121-138 (2019).

Koley, S., Mondal, R., and Sahoo, T., "Fredholm integral equation technique for hydroelastic analysis of a floating flexible porous plate,’ European Journal of Mechanics - B/Fluids 67, 291305 (2018).

Lamas-Pardo, M., Iglesias, G., and Carral, L., "A review of very large floating structures (VLFS) for coastal and offshore uses," Ocean Engineering 109, 677-690 (2015).

Liu, Y. and Li, Y., “An alternative analytical solution for water-wave motion over a submerged horizontal porous plate,” Journal of Engineering Mathematics 69, 385-400 (2011).

Mahmood-Ul-Hassan, H., Meylan, M. H., and Peter, M. A., "Water-wave scattering by sub- 
merged elastic plates," The Quarterly Journal of Mechanics and Applied Mathematics 62, 321$344(2009)$.

Martin, P. and Farina, L., "Radiation of water waves by a heaving submerged horizontal disc," Journal of Fluid Mechanics 337, 365-379 (1997).

Meylan, M. H., "Wave response of an ice floe of arbitrary geometry," Journal of Geophysical Research 107, 3005 (2002).

Meylan, M. H., Bennetts, L. G., and Peter, M. A., "Water-wave scattering and energy dissipation by a floating porous elastic plate in three dimensions," Wave Motion 70, 240-250 (2017).

Meylan, M. H. and Squire, V. A., "Response of a circular ice floe to ocean waves," Journal of Geophysical Research 101, 8869-8884 (1996).

Mohapatra, S. C., Sahoo, T., and Guedes Soares, C., "Surface gravity wave interaction with a submerged horizontal flexible porous plate,” Applied Ocean Research 78, 61-74 (2018).

Porter, R., "Linearised water wave problems involving submerged horizontal plates," Applied Ocean Research 50, 91-109 (2015).

Porter, R. and Evans, D., "Complementary approximations to wave scattering by vertical barriers," Journal of Fluid Mechanics 294, 155-180 (1995).

Press, W. H., Teukolsky, S. A., Vetterling, W. T., and Flannery, B. P., Numerical recipes: The art of scientific computing (Cambridge University Press, 2007).

Renzi, E., "Hydroelectromechanical modelling of a piezoelectric wave energy converter,' Proceedings of the Royal Society A: Mathematical, Physical and Engineering Sciences 472, 20160715 (2016).

Sahoo, T., Lee, M., and Chwang, A., "Trapping and generation of waves by vertical porous structures," Journal of Engineering Mechanics 126, 1074-1082 (2000).

Sahoo, T., Yip, T. L., and Chwang, A. T., "Scattering of surface waves by a semi-infinite floating elastic plate," Physics of Fluids 13, 3215 (2001).

Squire, V. A., "Synergies between VLFS hydroelasticity and sea-ice research," in The Eighteenth International Offshore and Polar Engineering Conference (International Society of Offshore and Polar Engineers, 2008) pp. 1-13.

Squire, V. A., "Ocean wave interactions with sea ice: a reappraisal," Annual Review of Fluid Mechanics 52, 37-60 (2020).

Thompson, I., Linton, C., and Porter, R., "A new approximation method for scattering by long finite arrays," The Quarterly Journal of Mechanics and Applied Mathematics 61, 333-352 (2008). 
Williams, T. D. and Meylan, M. H., “The Wiener-hopf and residue calculus solutions for a submerged semi-infinite elastic plate," Journal of Engineering Mathematics 75, 81-106 (2012).

Yu, X. and Chwang, A. T., "Analysis of wave scattering by submerged circular disk," Journal of Engineering Mechanics 119, 1804-1817 (1993).

Yu, X. and Chwang, A. T., "Water waves above submerged porous plate," Journal of Engineering Mechanics 120, 1270-1280 (1994).

Zhao, C., Zhang, J., and Huang, W., "Vibration reduction of floating elastic plates in water waves," Marine Structures 20, 71-99 (2007).

Zheng, S., Antonini, A., Zhang, Y., Greaves, D., Miles, J., and Iglesias, G., "Wave power extraction from multiple oscillating water columns along a straight coast," Journal of Fluid Mechanics 878, 445-480 (2019).

Zheng, S., Meylan, M. H., Fan, L., Greaves, D., and Iglesias, G., "Wave scattering by a floating porous elastic plate of arbitrary shape: a semi-analytical study," Journal of Fluids and Structures 92, 102827 (2020).

Zheng, S. and Zhang, Y., "Wave diffraction from a truncated cylinder in front of a vertical wall," Ocean Engineering 104, 329-343 (2015).

Zheng, S. and Zhang, Y., "Wave radiation from a truncated cylinder in front of a vertical wall," Ocean Engineering 111, 602-614 (2016).

Zheng, S. and Zhang, Y., "Theoretical modelling of a new hybrid wave energy converter in regular waves," Renewable Energy 128, 125-141 (2018).

Zheng, S., Zhang, Y., and Iglesias, G., "Wave-structure interaction in hybrid wave farms," Journal of Fluids and Structures 83, 386-412 (2018).

Zheng, S., Zhang, Y., and Iglesias, G., "Coast/breakwater-integrated OWC: A theoretical model," Marine Structures 66, 121-135 (2019). 